\title{
"تخطيط الحملات الإعلانية المعاصرة بين الفلسفة الرمزية والإبداع البصري الرقمي" \\ "Planning Contemporary Advertising Campaigns between Symbolic Philosophy and Digital Visual Innovation"
}

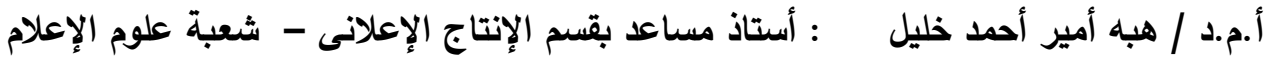

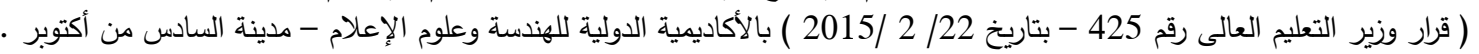

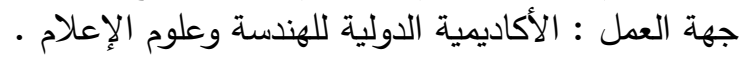 \\ الدرجة العلية : دكتوراه الفلسفة فى الفنون التطبيقية - قسم الإعلان - كلية الفنون التطبيقية - جامعة حلوان.
}

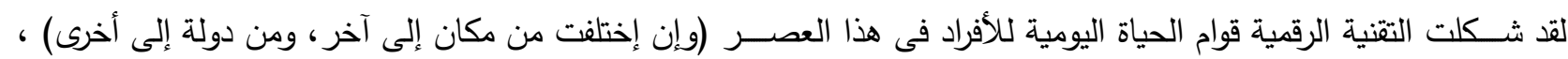

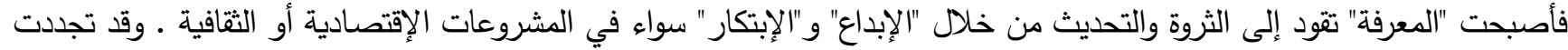

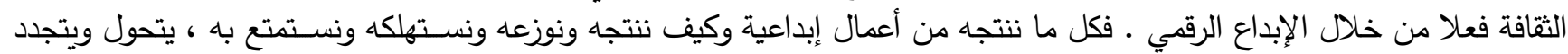

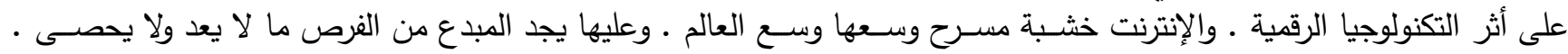

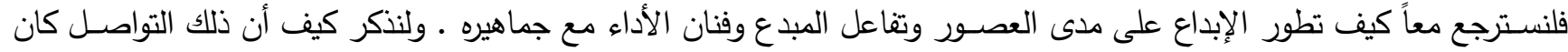

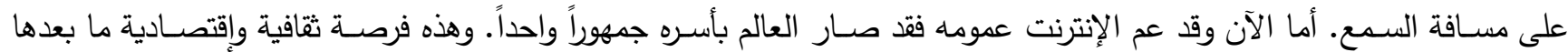

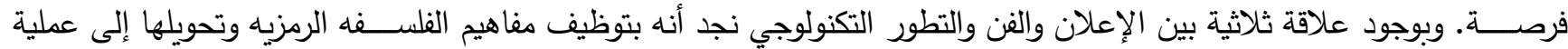

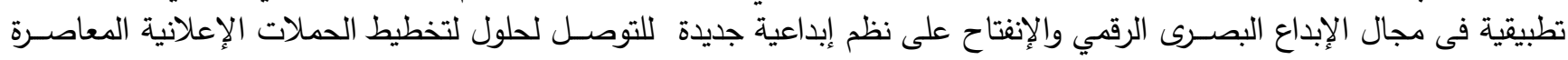

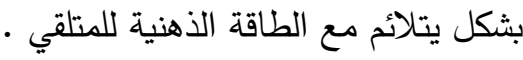
مفاهيم ذات مالة بموضموع البحث:

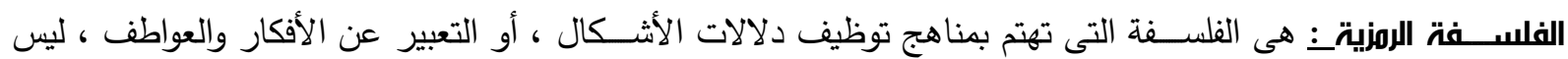

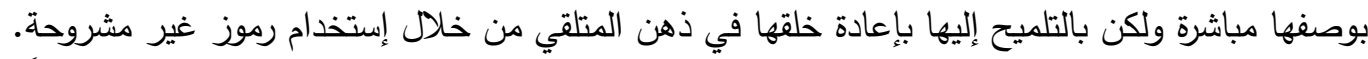

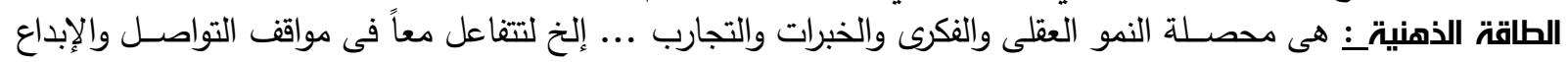
فهى مقدرة ذهن الشخص للتفسير والإدراك للتفاعل وعمل نشاط خارجى.

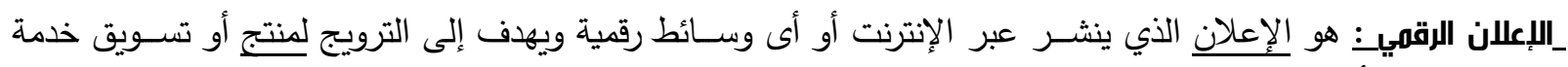

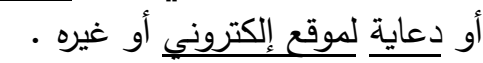

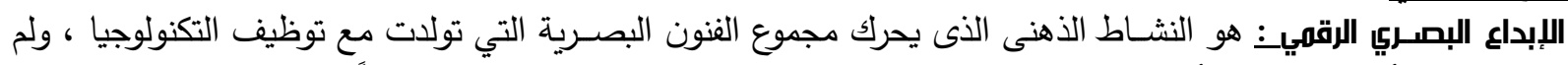
تكن موجودة قبل ذلك ، أو تطورت من أشكال قديمة ، ولكنها إتخذت مع إستخدام التكنولوجيا الرقمية صوراً جديدة في الإنتاج والتلقي تلوني

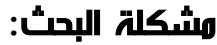

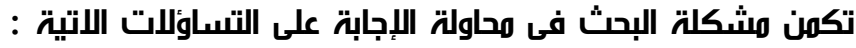
1) إلى أى مدى يمكن الإستفادة من الفلسفة الرمزية لتحقيق الإبداع البصرى الرقمى فى الرئ تخطيط الحملات الإعلانية؟

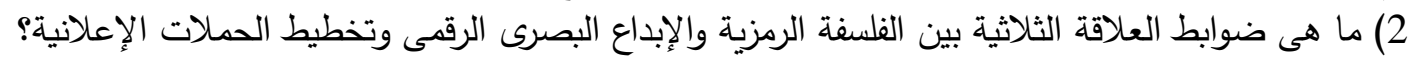

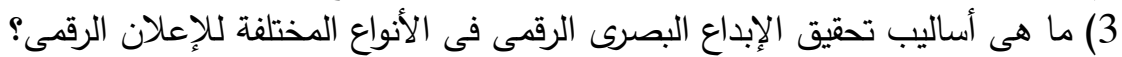

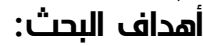
يهدف البحث إلى: البه: 1) الإســتفادة من المفاهيم الخاصـــة بالفلسـفـة الرمزية لفتح أفاقاً جديدة للإبداع البصــرى الرقمى لتخطيط حملة إعلانية تلائم روح

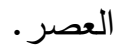
2) إيجاد معايير فنية إبداعية للإعلان الرقمى بما يتلائم مع الطاقة الذهنية للمتلقى المستهدف.

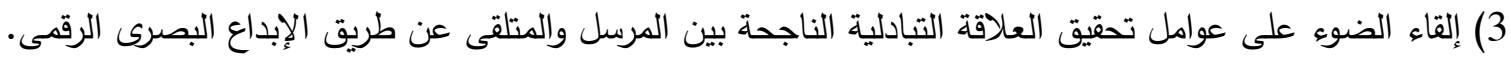

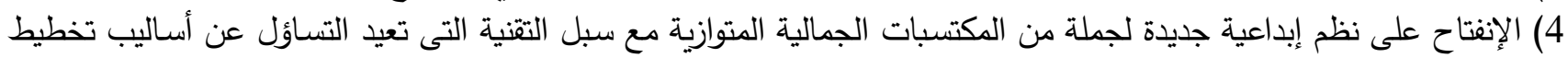
الحملات الإعلانية المعاصرة.

فروف البحث: يفترغ البحث أنه: 1) يمكن تحويل المفاهيم الفلهـفية النظرية إلى عملية تطبيقية فى تصـميم الإعلان لتمثل قيمة معرفية جديدة عن طريق أفكار مبتكرة يستثفها المصدم من دراسته الفلسفية. 2) بإيقاعات العصر الجديدة تغيرت مفاهيم الإبداع فأصبحت مرتبطة بالتتنية. 
3) إســتثمار المصــمم لتقنيات التكنولوجيا المختلفة يعمل على تطوير المخيلة على الإبتكار فى المنحى الإبداعى لأســاليب تخطيط

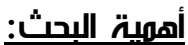

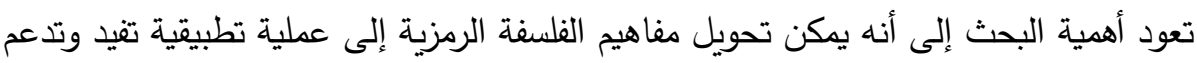

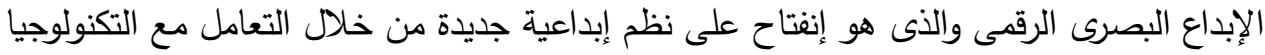

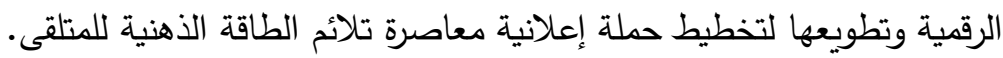

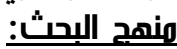

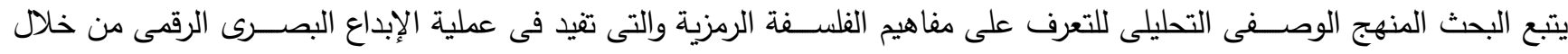

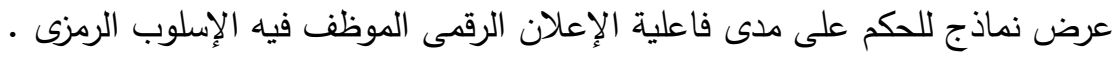

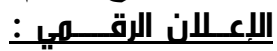

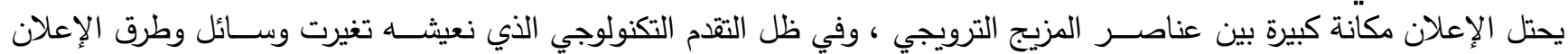

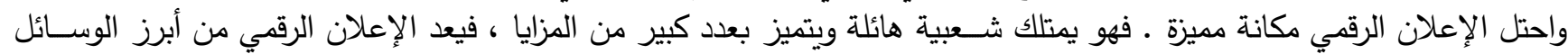

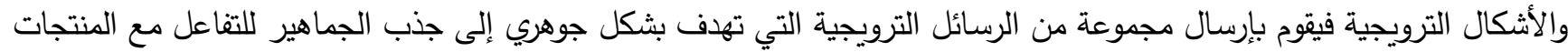

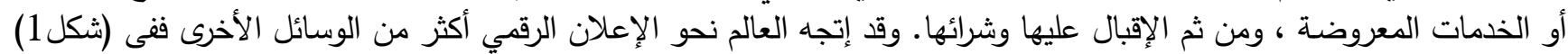

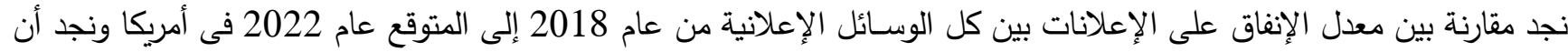

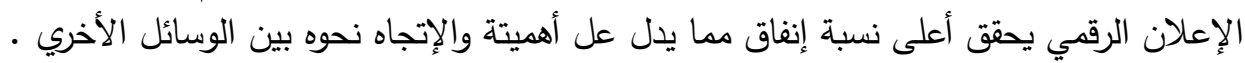

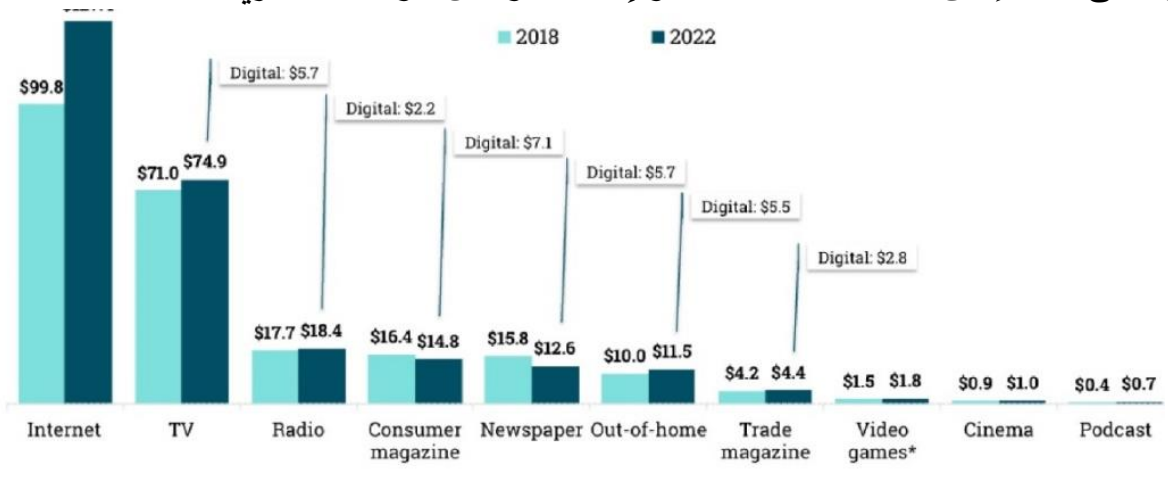

US Advertising Media Market sizes (\$B), 2018 v. 2022

مقارنة بين معدل الإنفاق على الإعلانات بين كل الوسائل فن الوقت الحالى و المتوقع فى r.rr

شكل (1) (22)

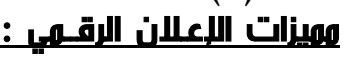

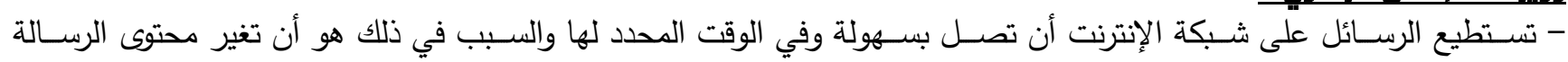

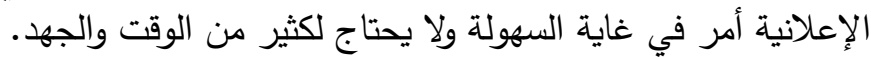

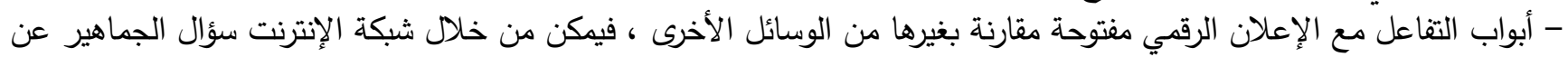

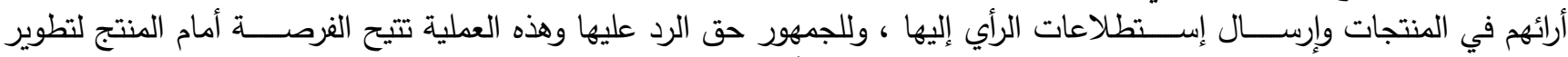

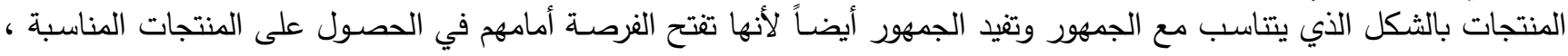
والتي تلبي إحتياجاتهم وتثبعها. - يتميز الإعلان الرقمي بإنخفاض تكلفتها: مقارنة بغيره من الوسائل الإعلانية الأخرى. 


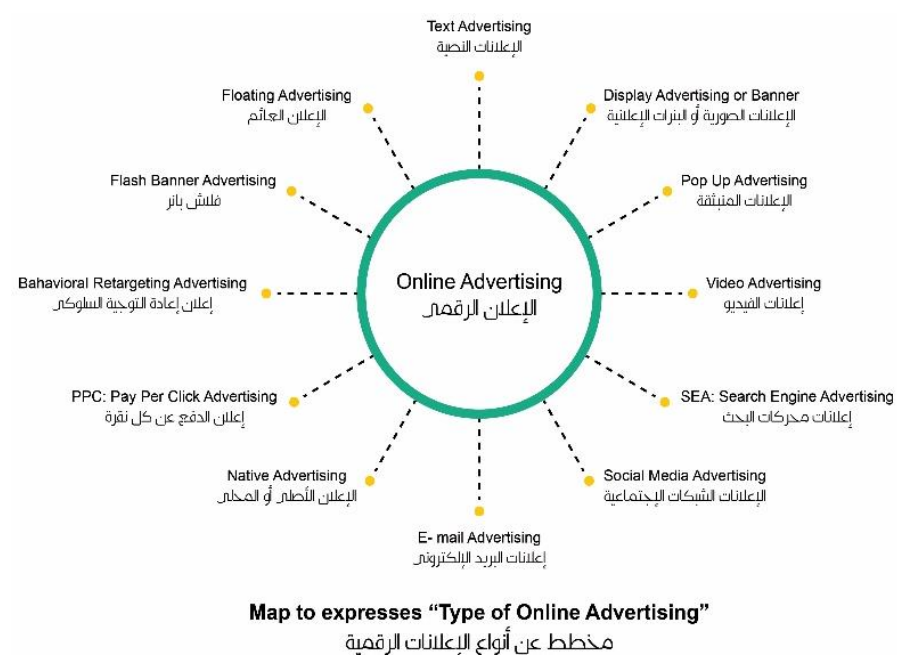
مخط عن أنواع البعلانات الرقمية

- يمكن القيام بتتفيذ إعلان لإستهاف شريحة معينة.

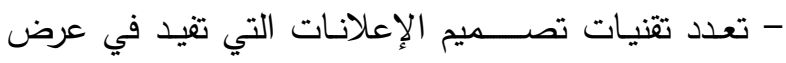
الإعلان بشكل مثير لجذب المتلقيين.

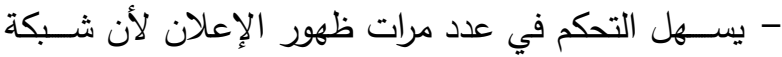

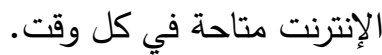

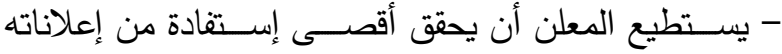

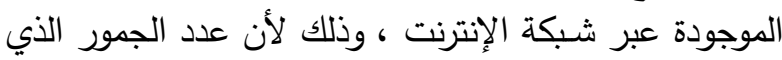

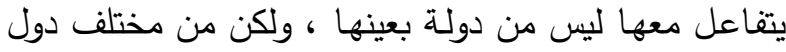

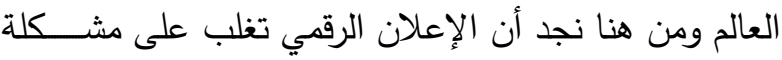

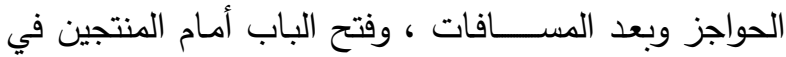

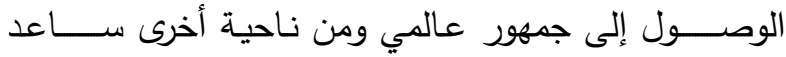

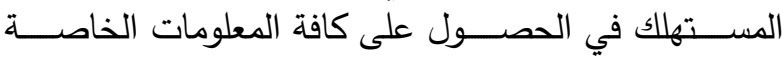
بالمنتجات والخدمات المعروضة.

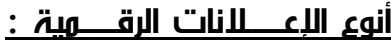
شكل (2)

:Text ads

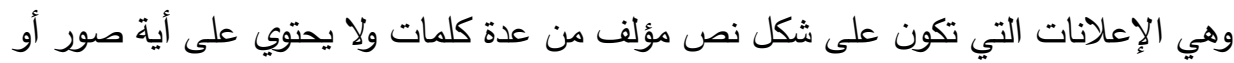

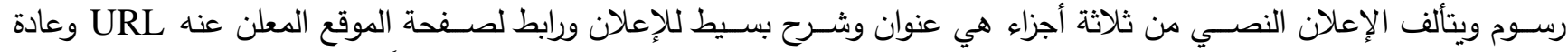

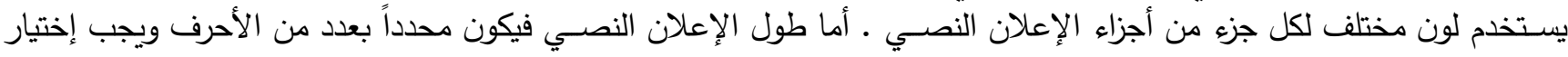
كلمات الإعلان النصي بدقه حتى تحفز الزائر للنقر على الإعلان النصي.

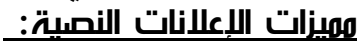

- رخيص التكلفة بالمقارنة ببقية أنواع الإعلان الرقمي.

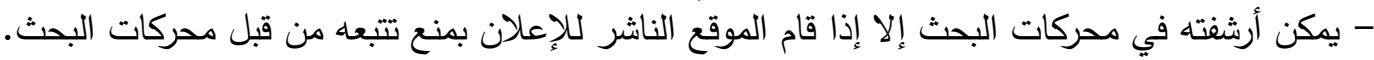

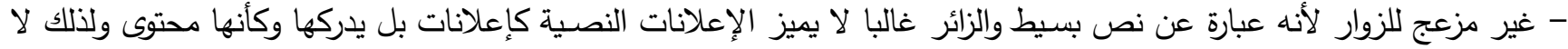
يتجاهلها وهذا ما يحقق الفائدة للموقع المعلن.

سلبيات الإعلانات النمية:

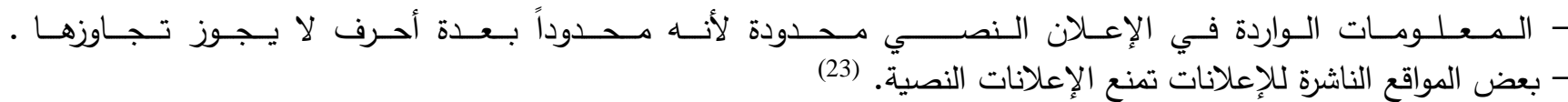

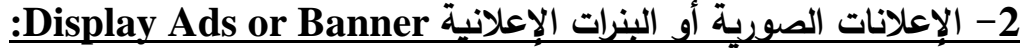

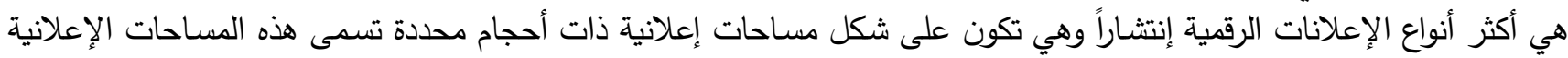

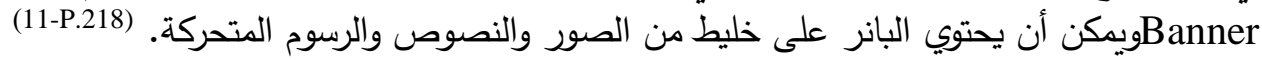

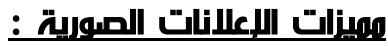

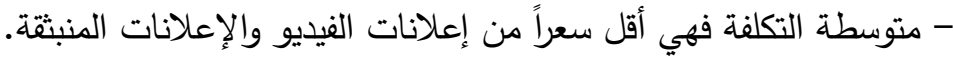

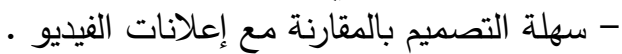

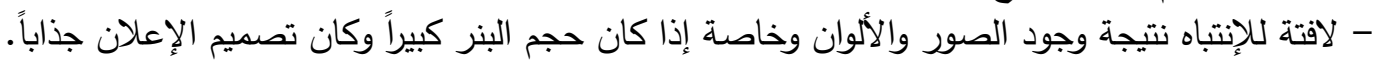

\section{سلبيات اللعالانات المورية:}

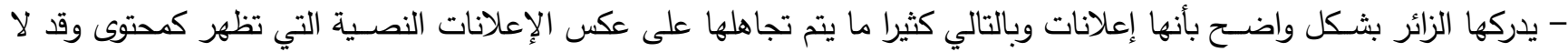
يدركها الزائر على إنها إعلانات.

- الحظر : هناك الكثير من البرامج التياتئي يستخدمها الزوار لحجبها.

:Pop up Ads

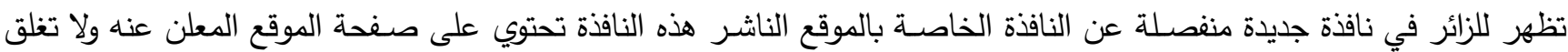

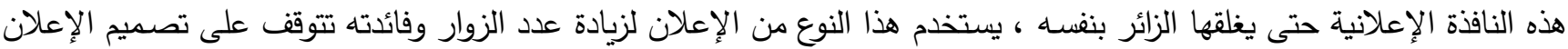

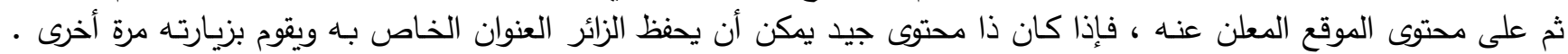

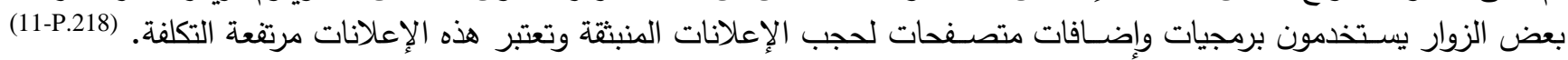




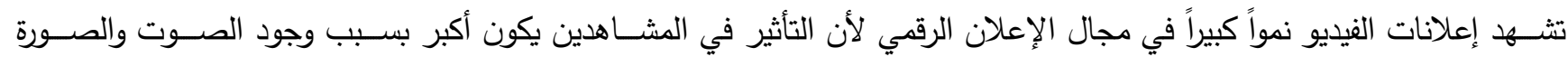

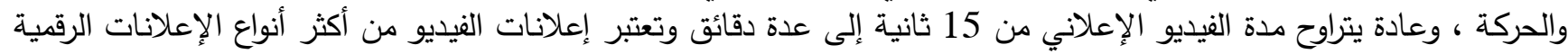

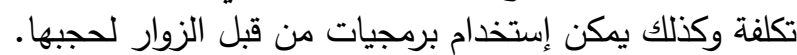

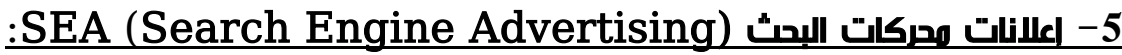

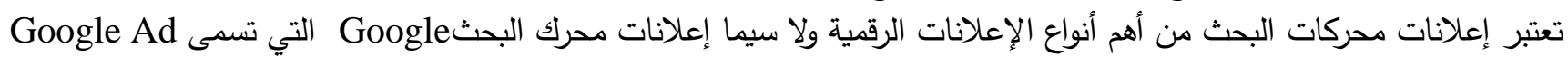

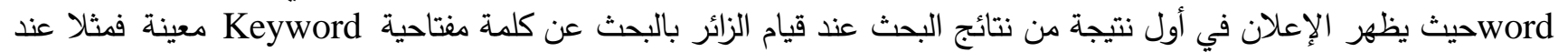

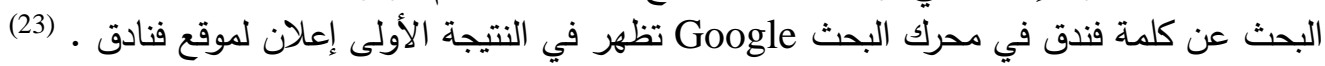

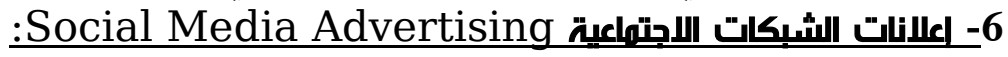

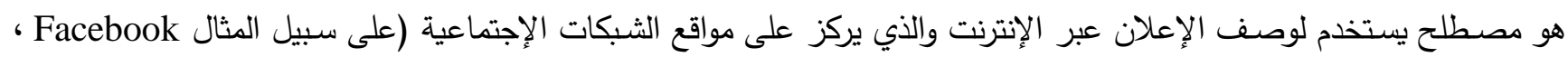

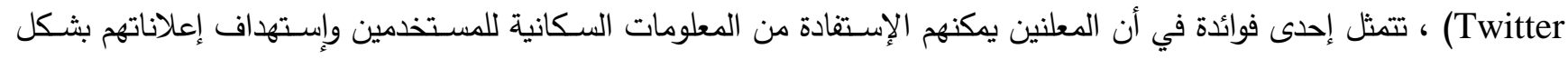

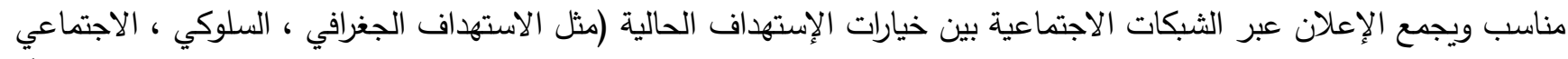

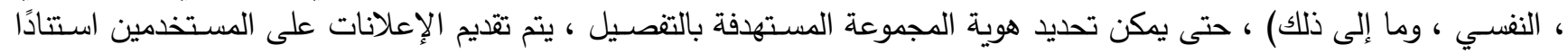

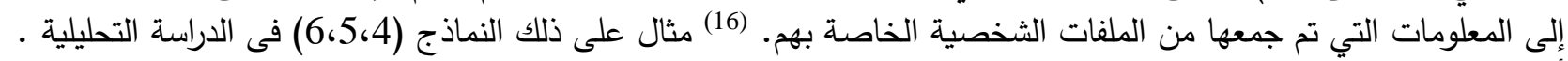

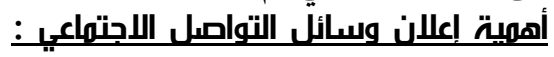

توليد العروف: يتم فيها ربط مواقع الثبكات الإجتماعية المنشورة بصفحة مقصودة وسيؤدي هذا الإرتباط إلى زيادة فاعلية الحملة.

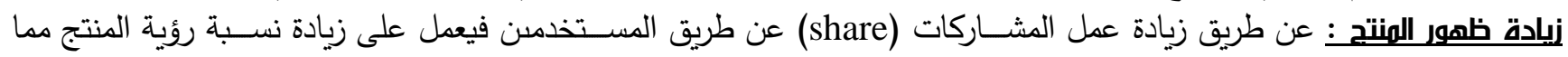

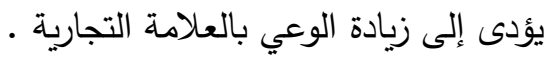

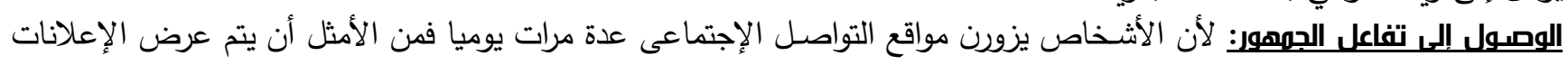

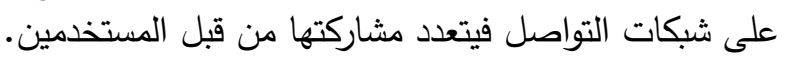

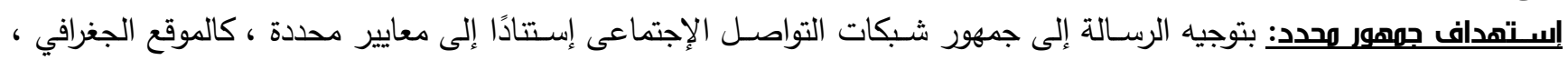

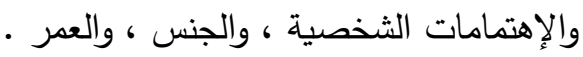
زيادة ولاء العملاء بالعلامة التجارية : وذلك لأنها تعطي الفرصة للتفاعل التهات المباشر مع العميل، مما يحافظ على الصورة الذهنية للنشاط

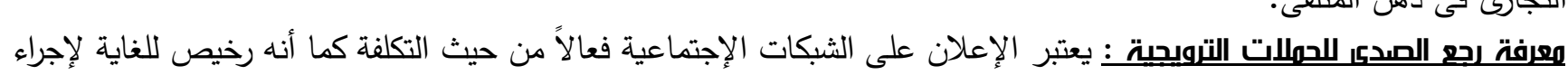

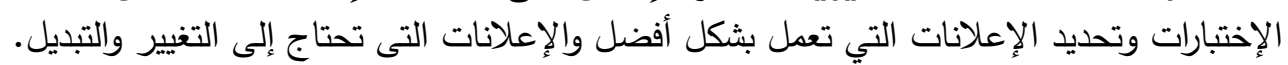

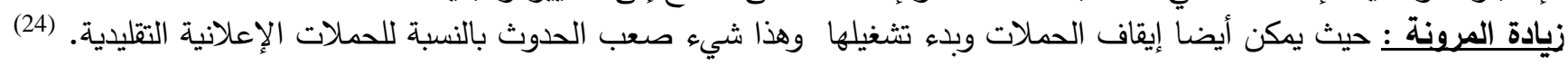

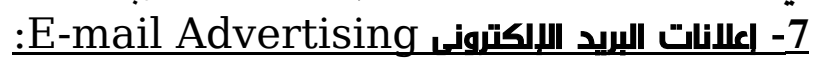

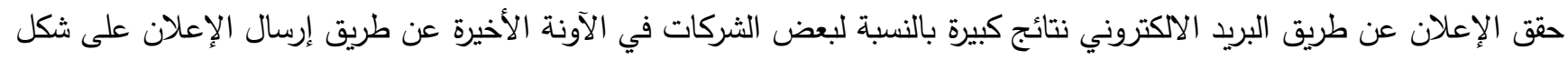

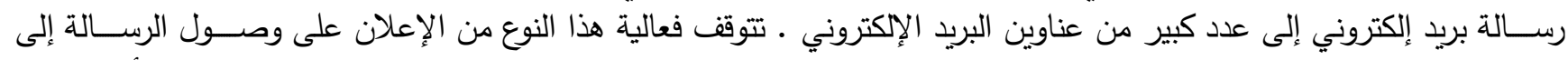

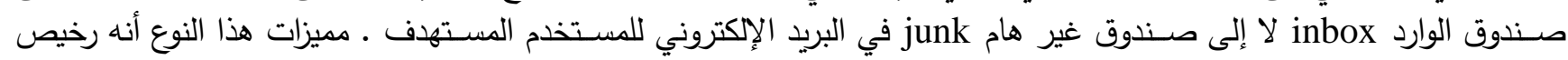

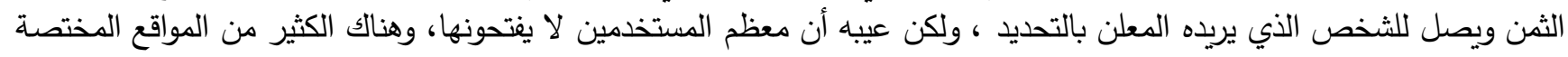

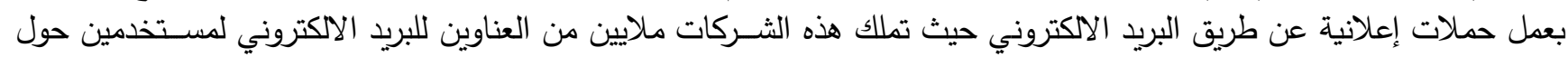

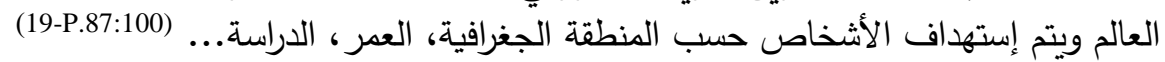

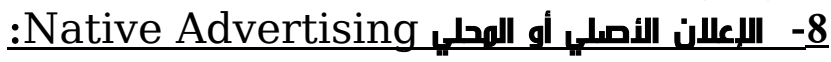

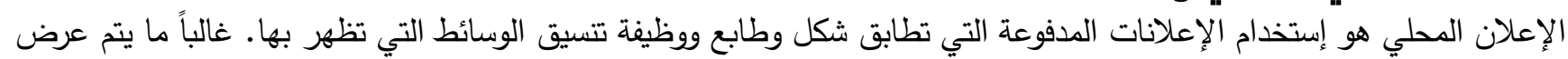

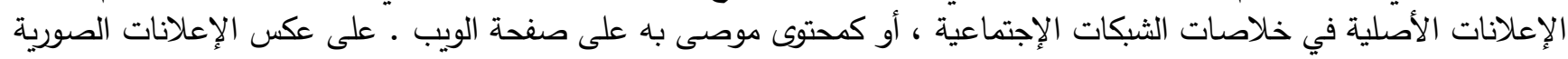

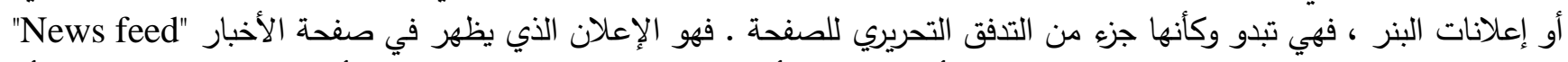

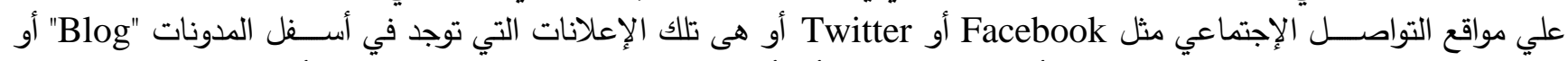

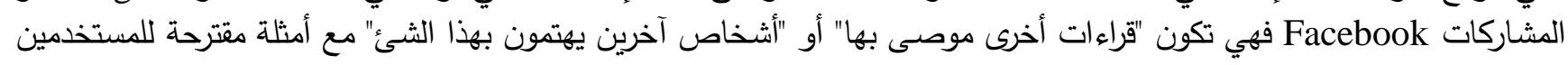

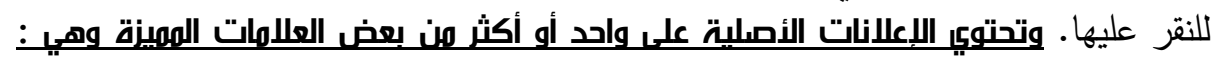

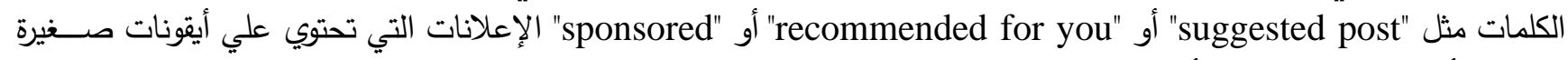
لإغلاقها أو حجب الإعلان إذا أراد المتلقي. 

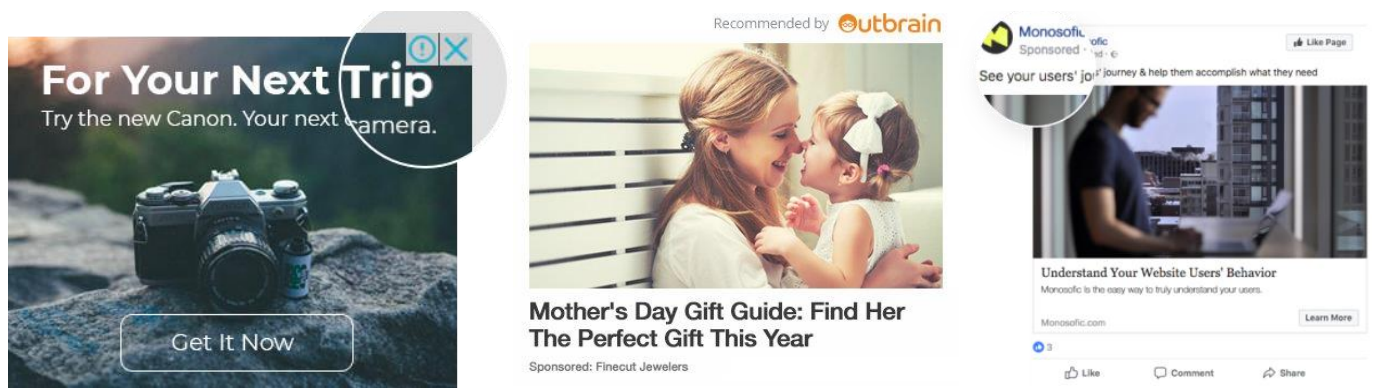

شكل (3) (25)

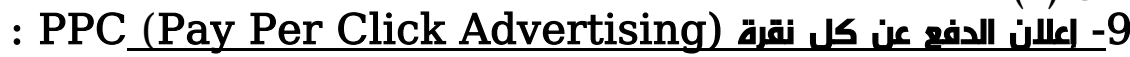

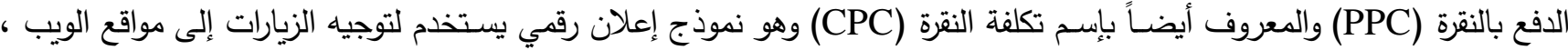

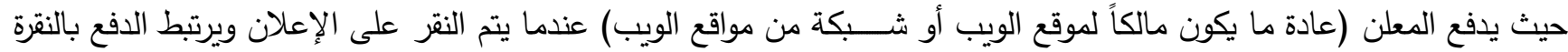

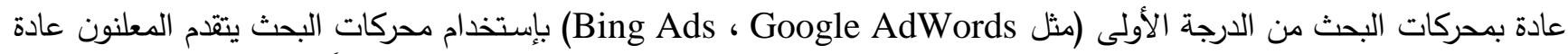

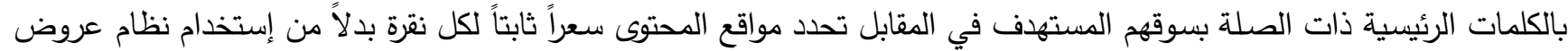

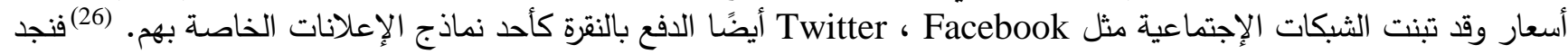

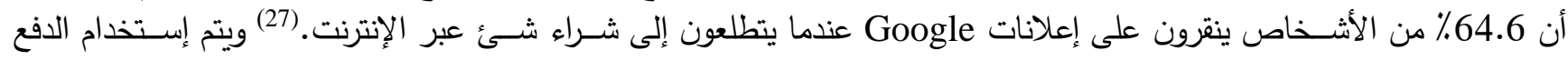

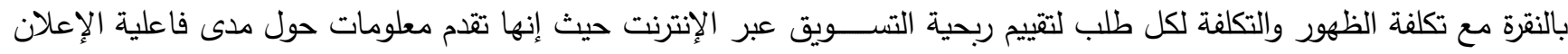

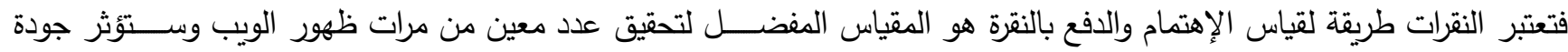

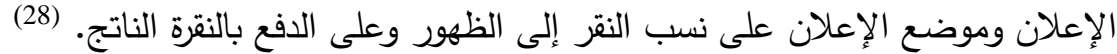

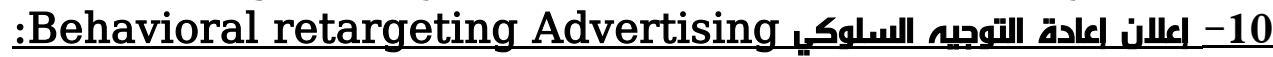

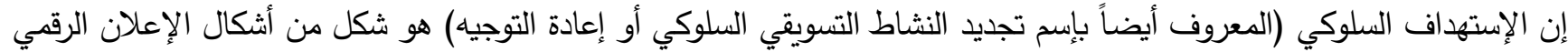

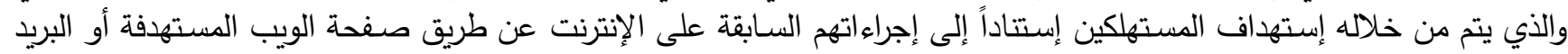

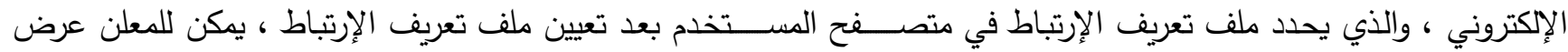

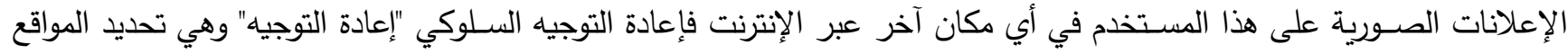

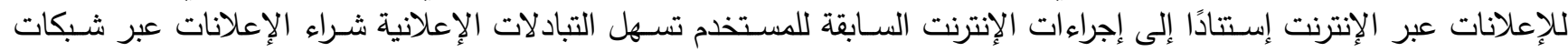

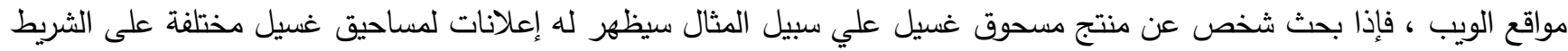

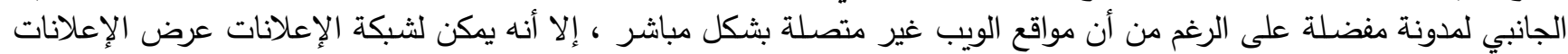

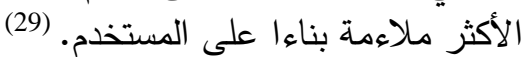

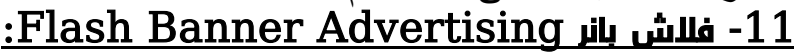

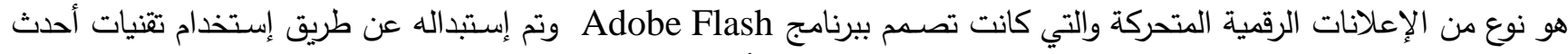
مثل ملفات GIF فهي توفر تجربة شـبيهة بالفيديو فعالة للغاية. حتي أنها سـهلة الإنثـاء (حتى إذا لم يكن لإي المصــمم الكثير من

المهارات التقنية). (30)

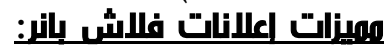

- لافتة للنظر

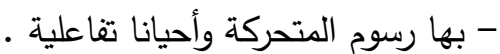
- يمكن أن تعرض القصص المنحكة واحيان لفاعدة نسبيا من خلال الرسوم المتحركة والكلمات .

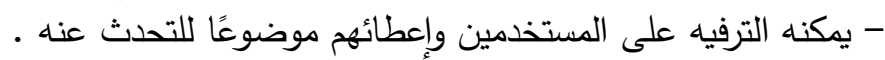

سلبيات اعلانتات فلاش بالنز:

- يجعل صفحة الويب أبطأ فى التحميل .

-12 يمكن أن تزعج المستخدمين إذا كانت إقتحامية ـ (31)

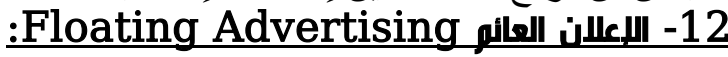

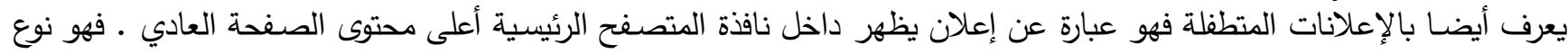

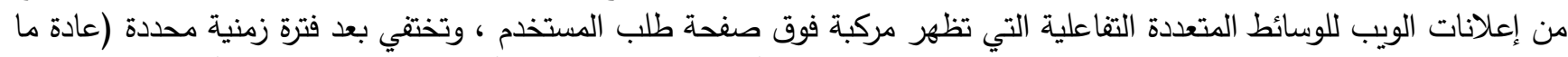

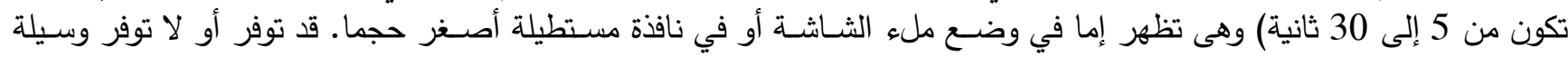

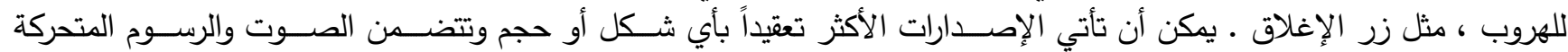

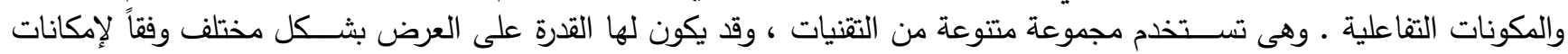

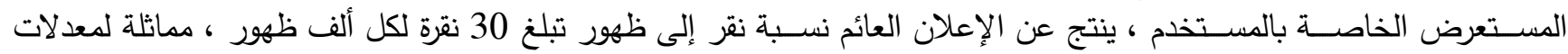




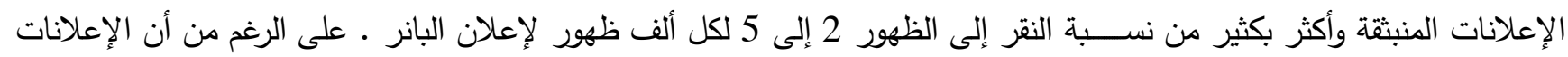

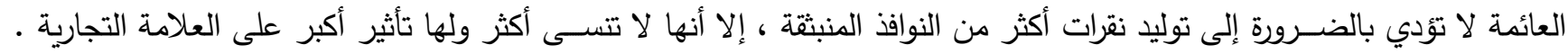

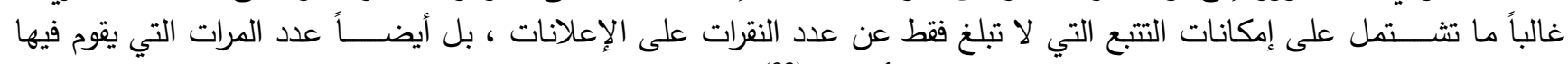
المستخدمون بإعادة تحميل الصفحة لمشاهدة الإعلان مرة أخرى.

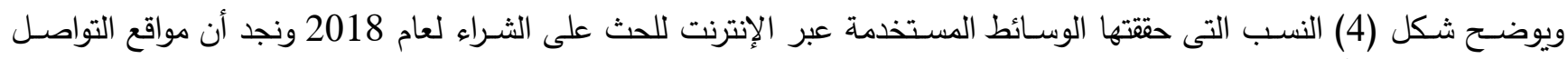

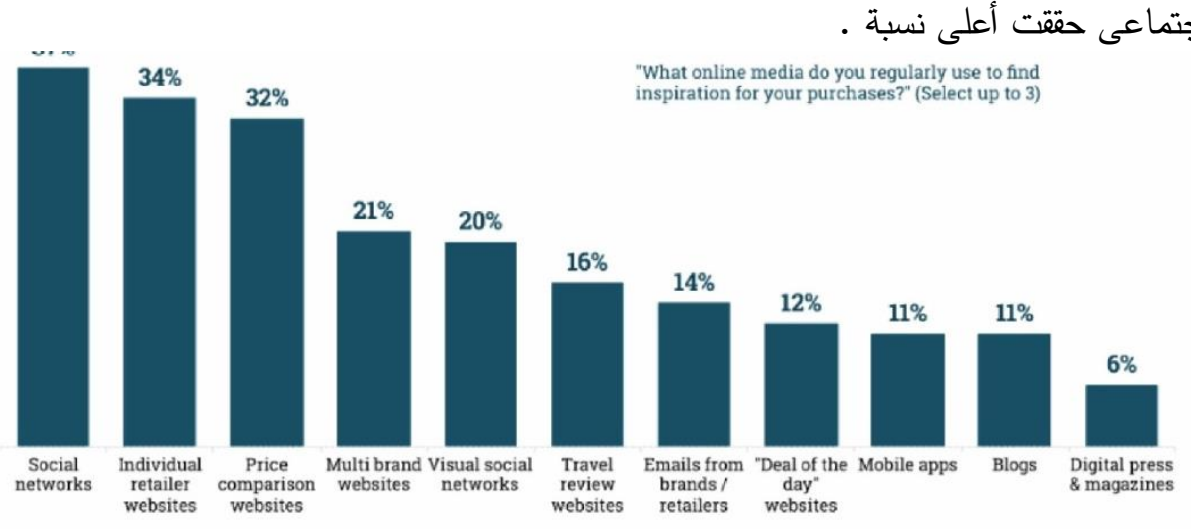

Online Media used for Purchase inspiration 2018

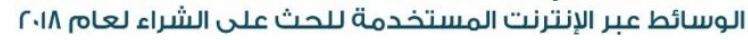

شكل (4) (3) (1)

الستخدار الروز في تمهيم اللعالان الرقهي:

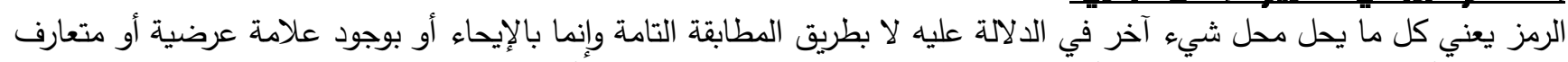

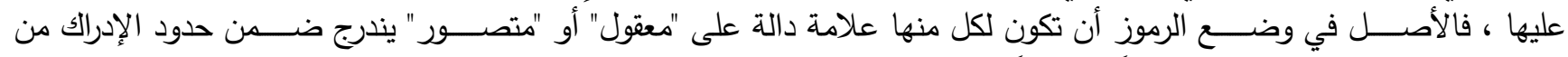

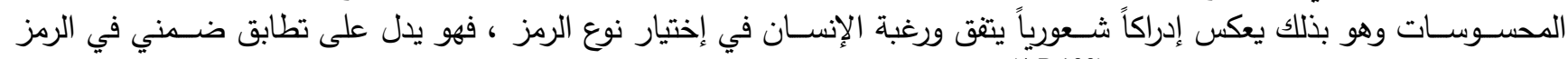

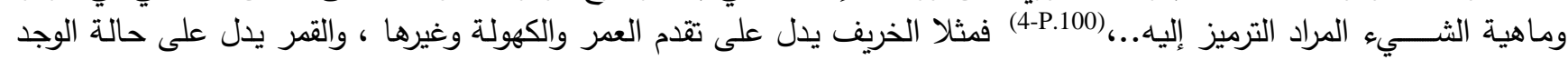

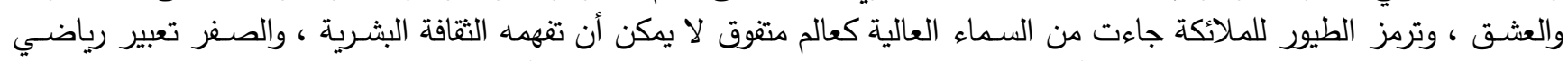

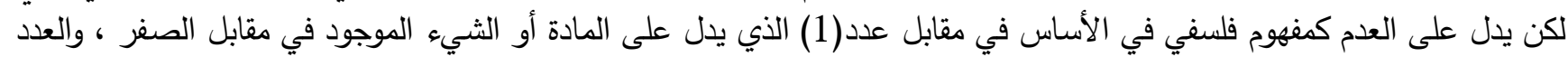

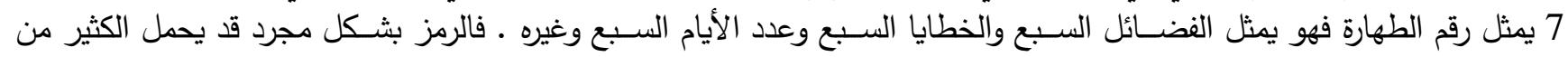

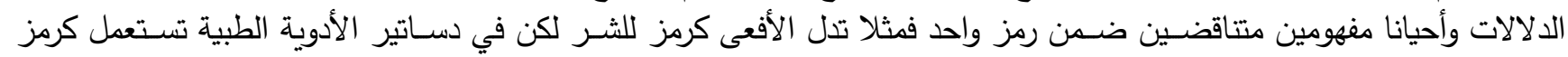

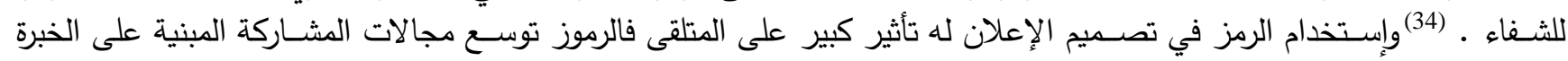

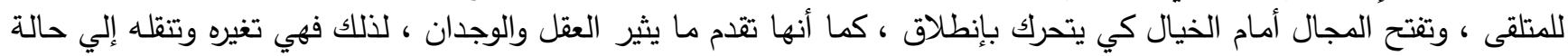

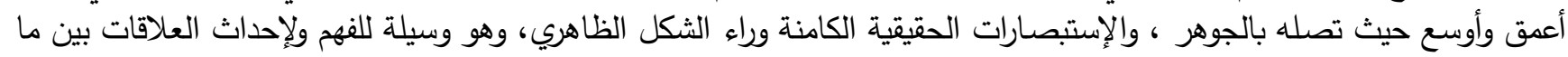

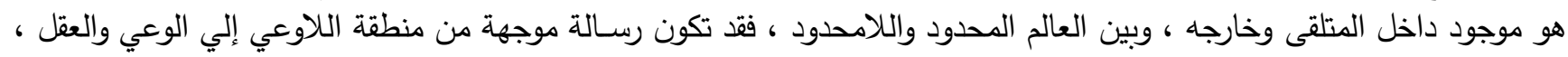

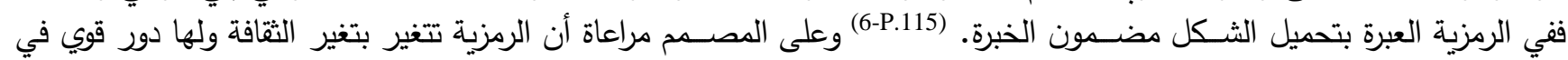

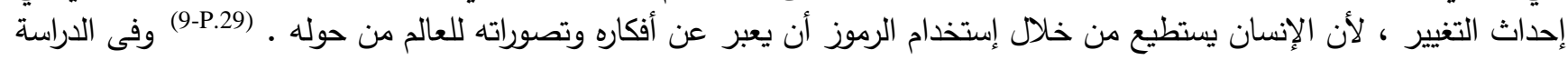

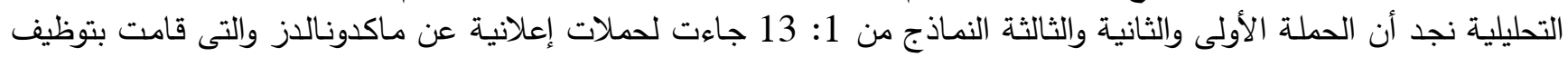

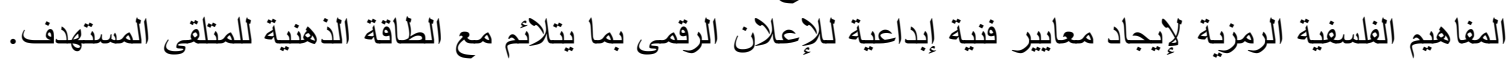

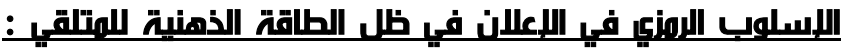

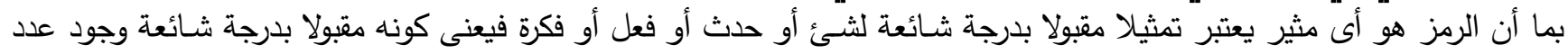

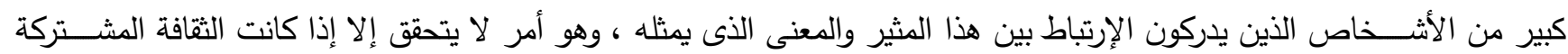

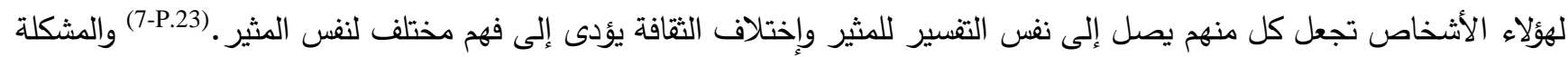

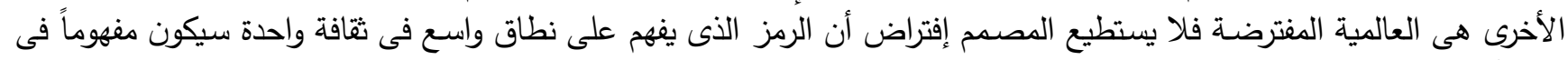

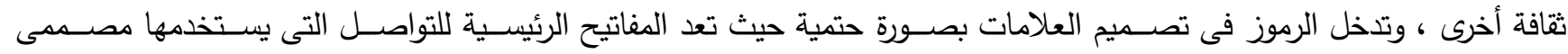

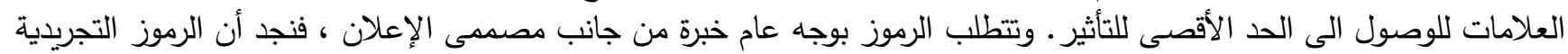

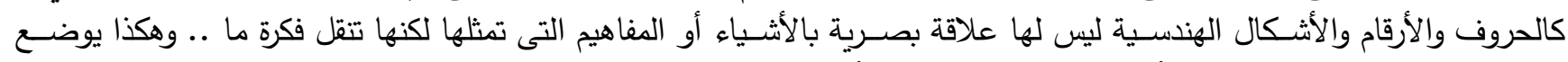

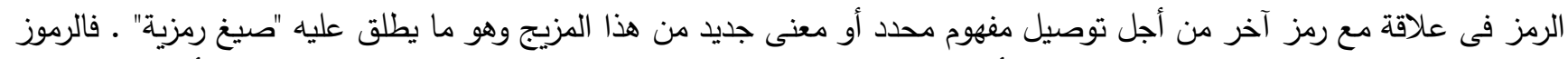

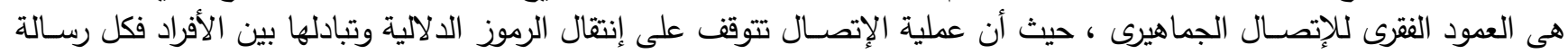

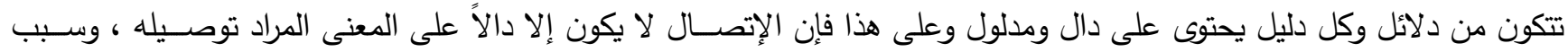




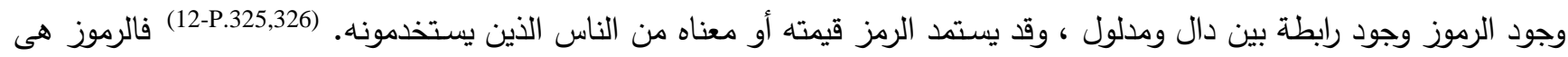

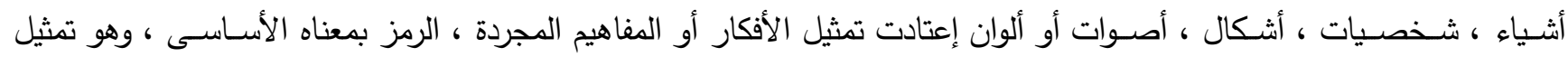

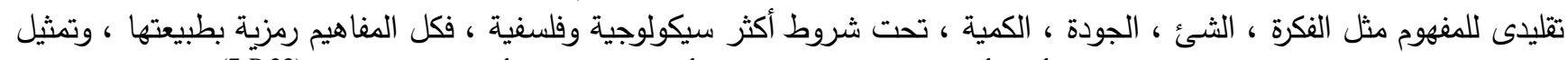

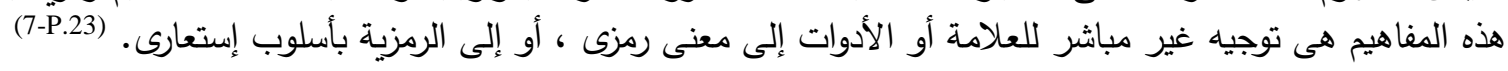

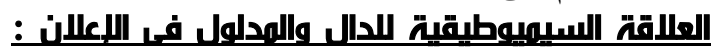

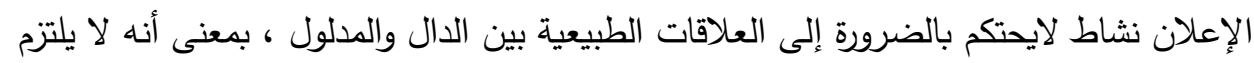

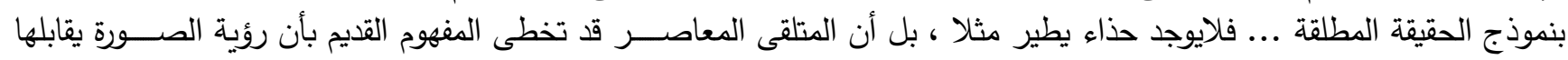

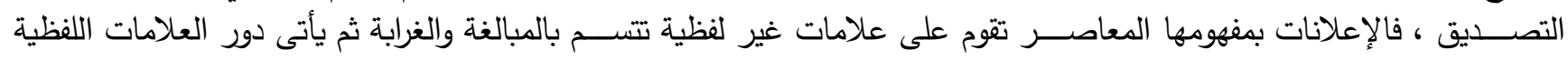

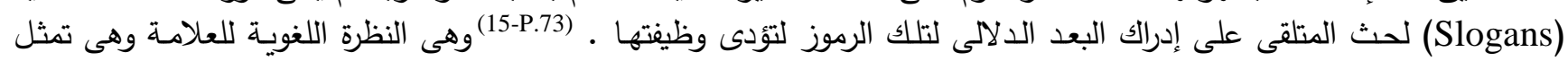

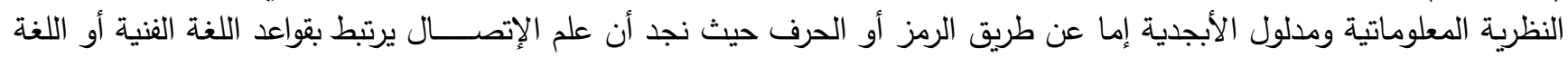

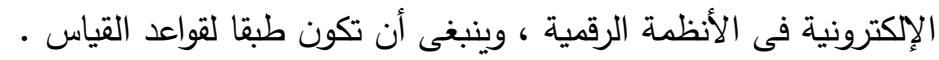

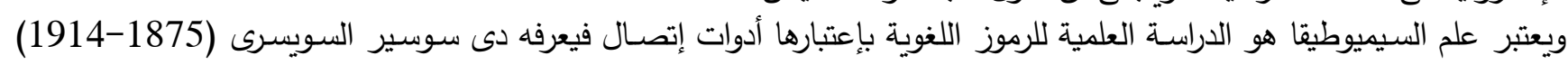

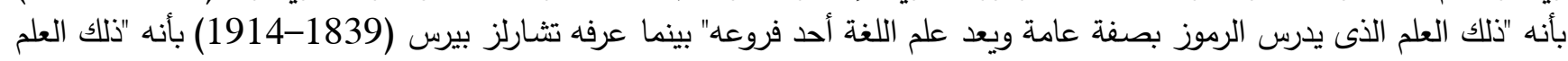

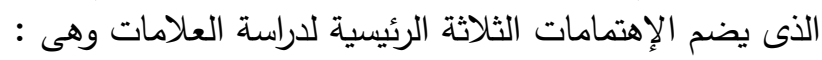

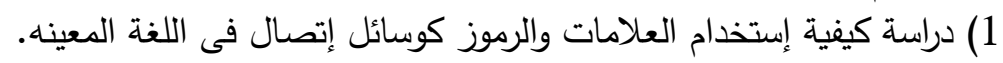
2) دراسة العلاقة بين الرمز وما يدل العلات علية أو يشير اليهاتيه.

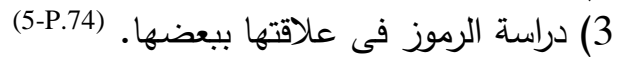

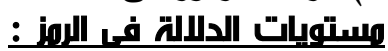

1

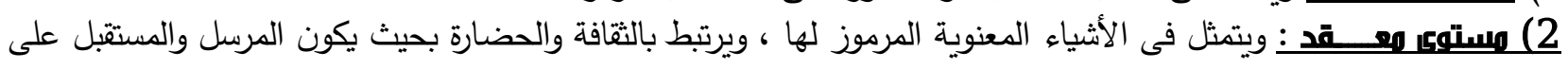

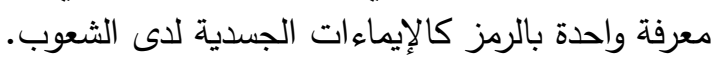

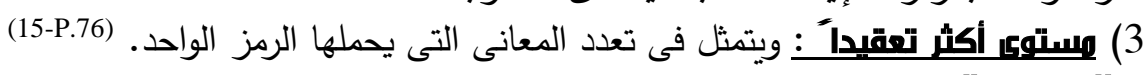

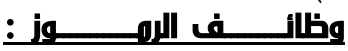

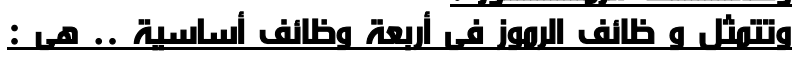

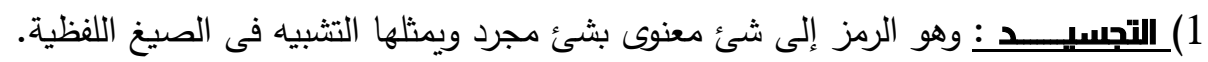

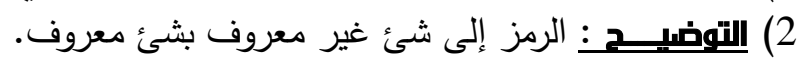

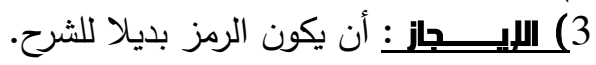

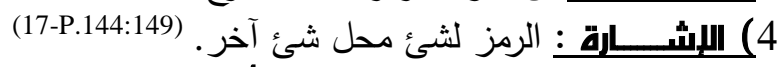

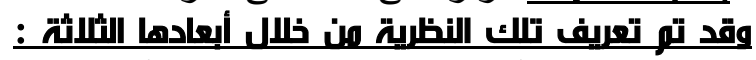

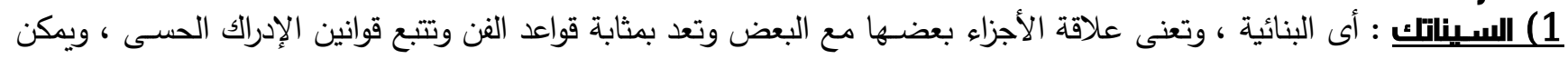

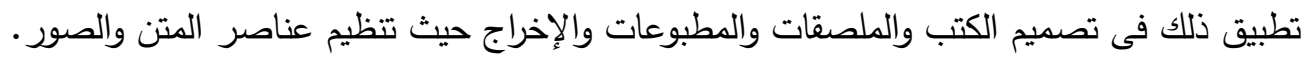

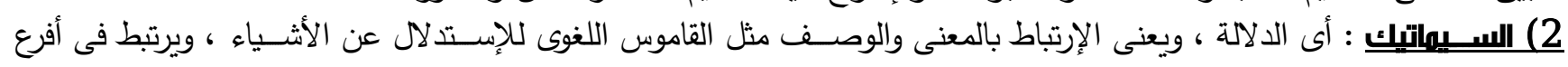

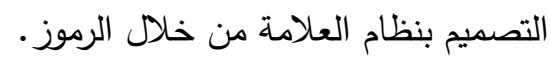
3) البرحماتك : وتعنى العملية والتجريب ، وتربط بين العلامة والإنتفاع بها أى الفرض منها حيث اللغة الفنية وإمكانية صــياغتها فى عناصر تجربيية. (14-P.247)

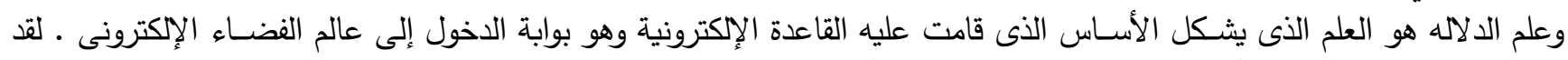

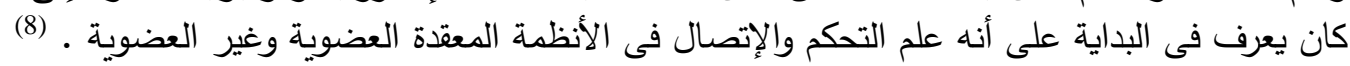

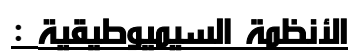

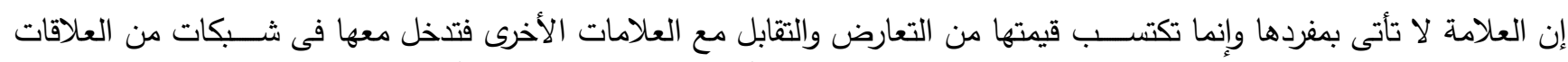

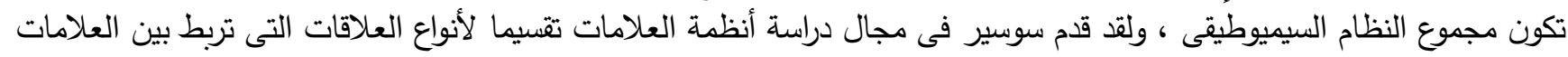

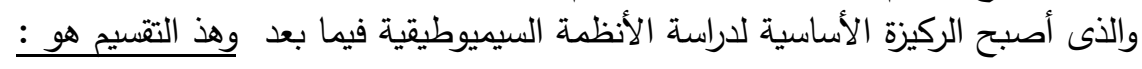

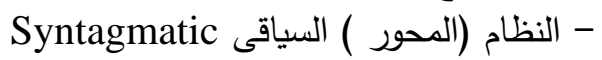

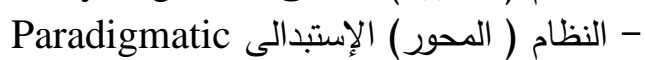

أولا : Syntagmatic النظار السياقئ)

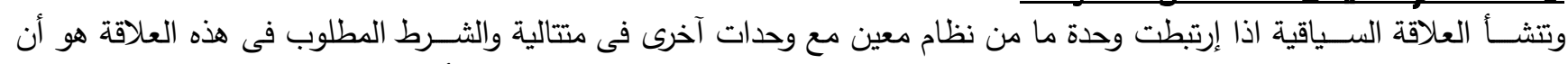

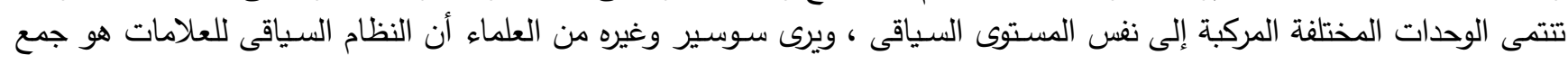




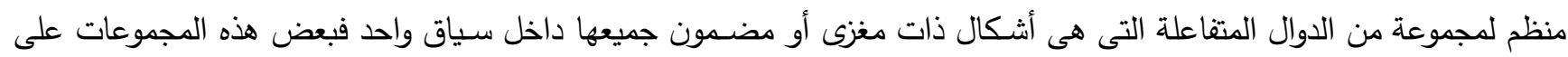

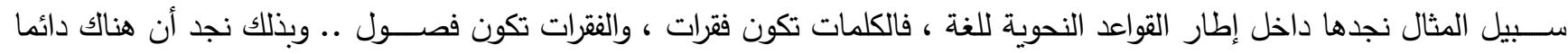

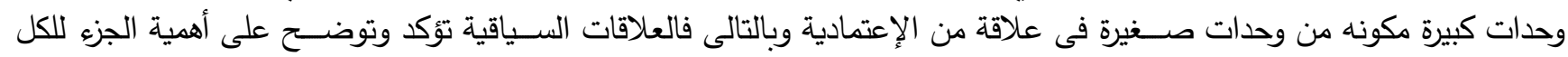

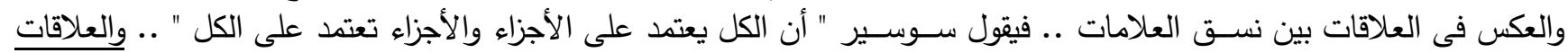

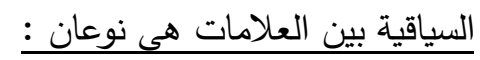

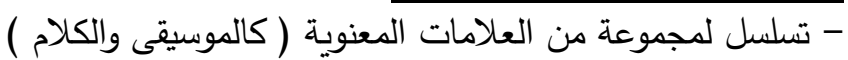

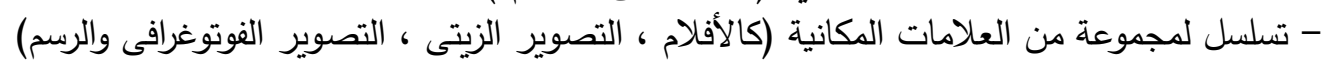

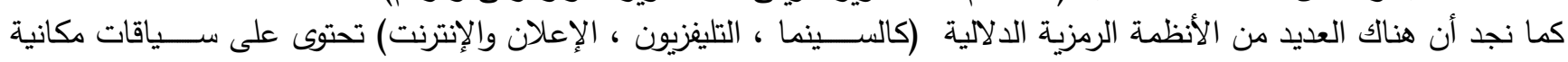

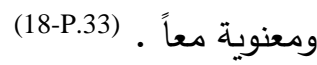

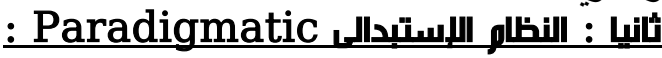

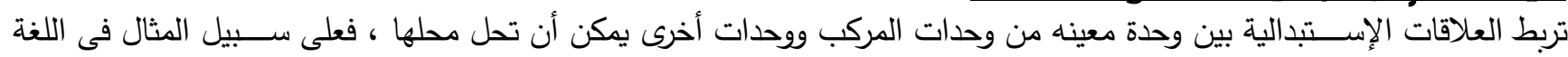

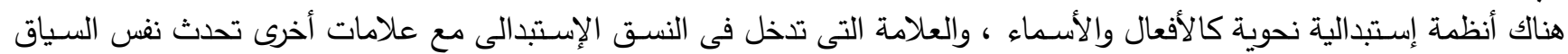

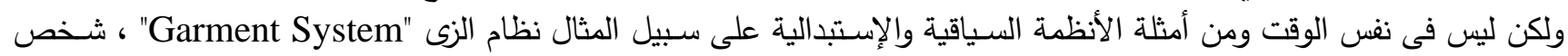

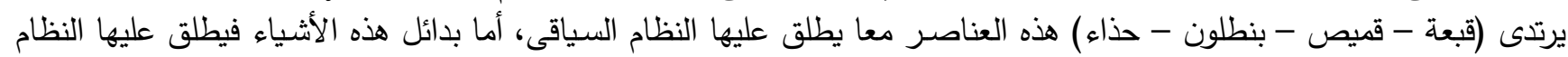

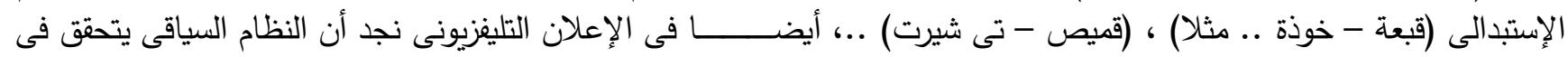

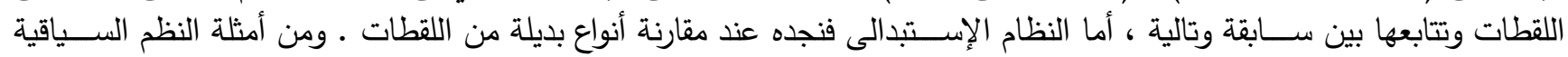

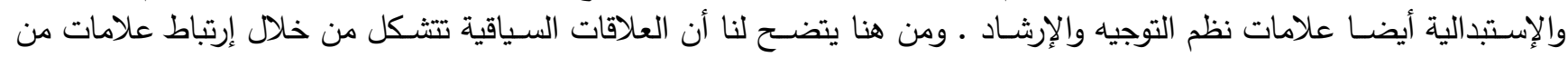

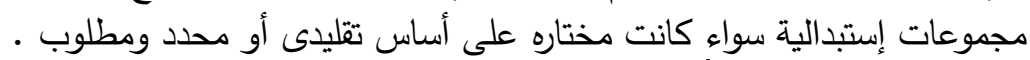

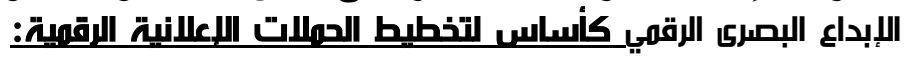

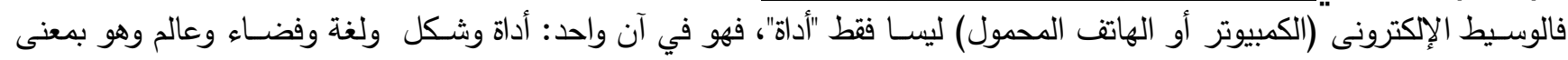

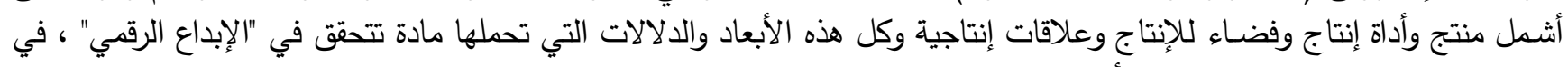

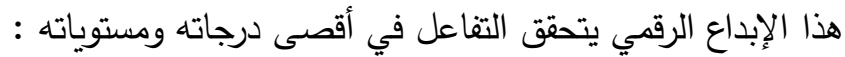

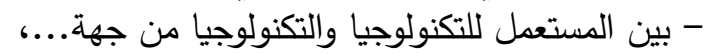

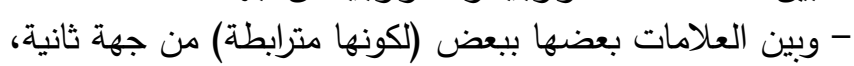

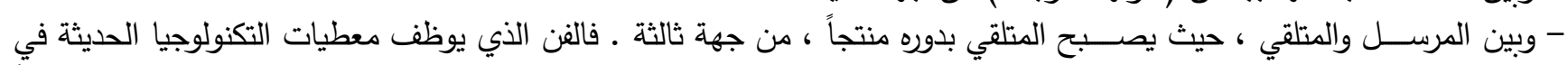

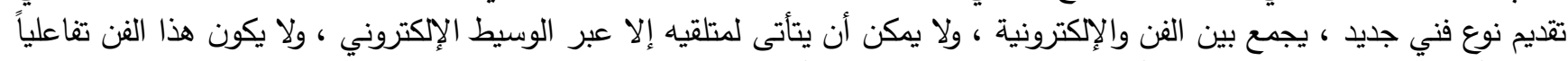

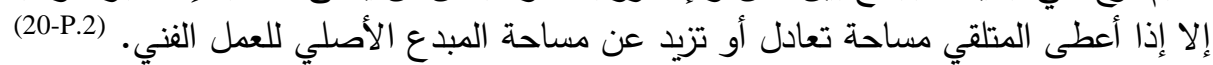
خمائم الإبداع البمركا الرقميا :

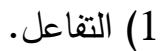
2) (1) الانفتاح. (1) التفرل. 3) التمركز حول المتلقي. (35) الإفتاح.

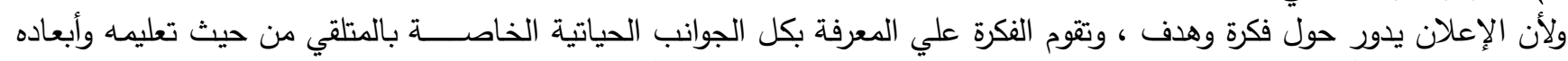

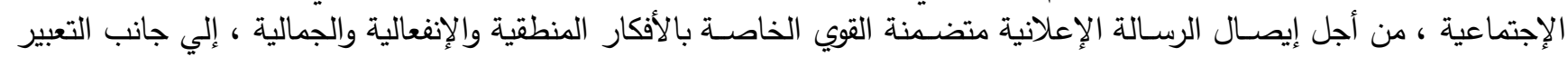

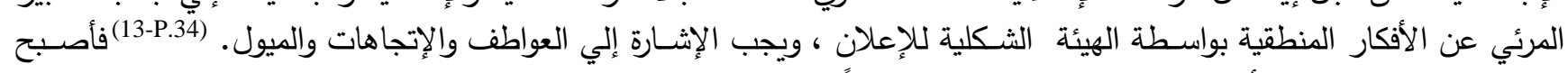

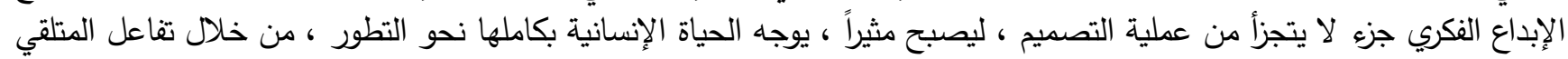

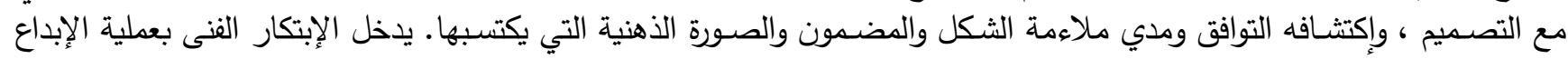

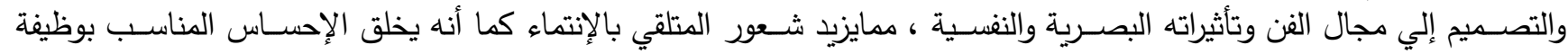

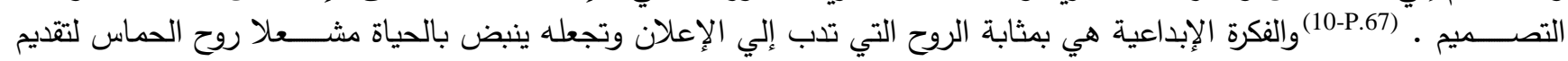

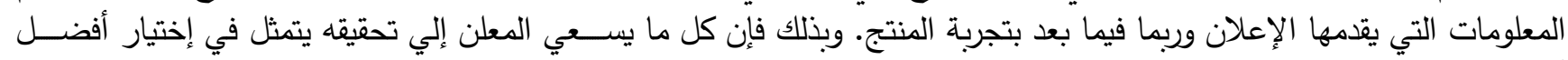

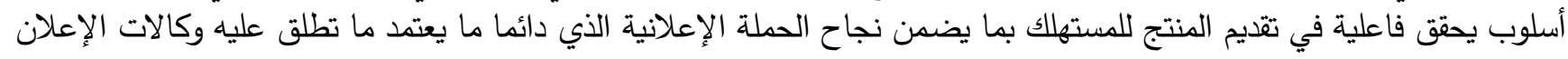

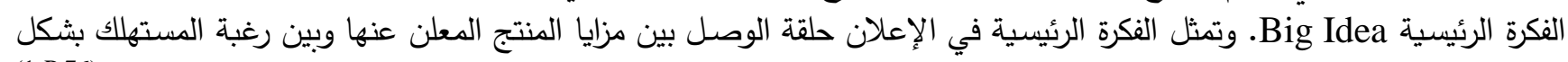

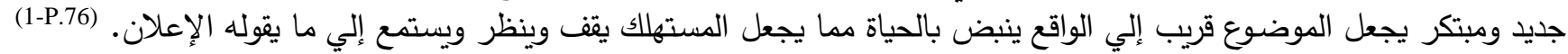

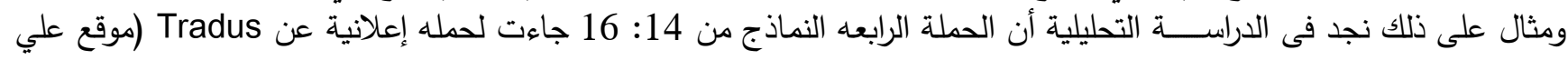

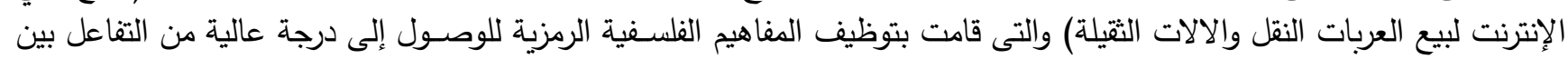


الأفكار الإعلانية الناجحه هي الأفكار ذات الوميض الإعلاني Flash of insight ، التي تدمج كل من مميزات المنتج المعلن عنه

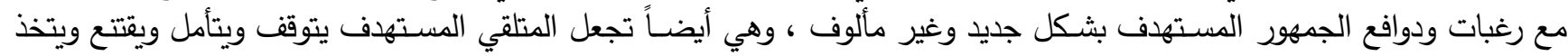

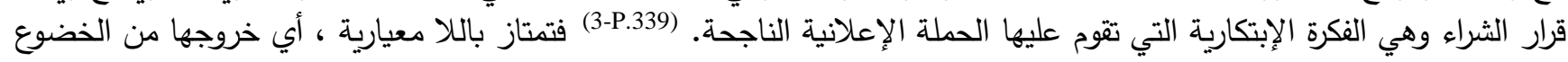

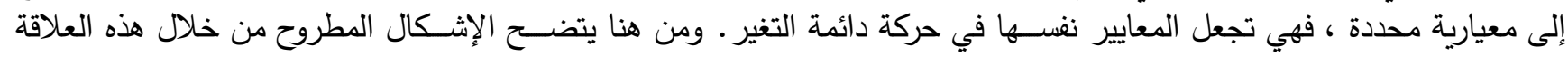

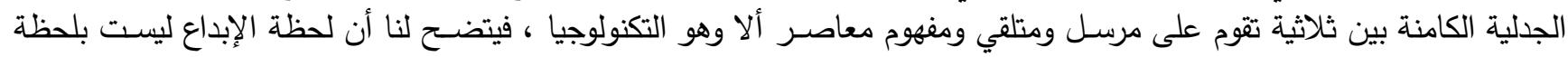

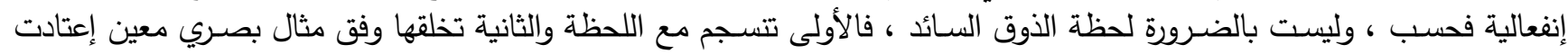

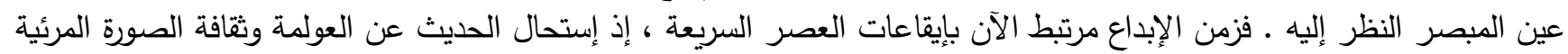

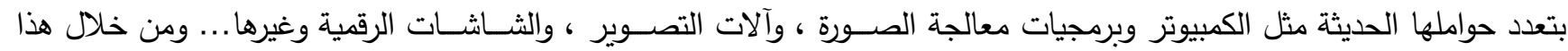

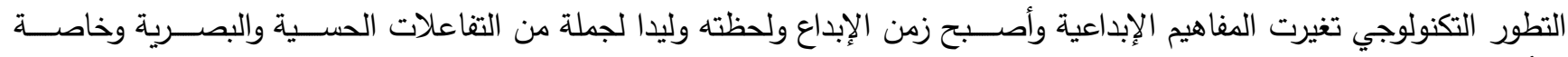

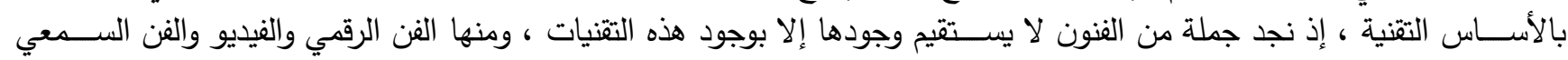

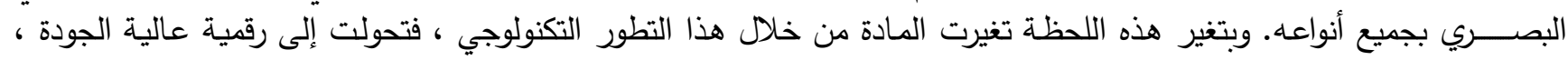

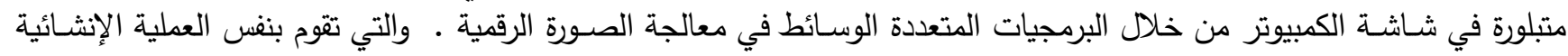

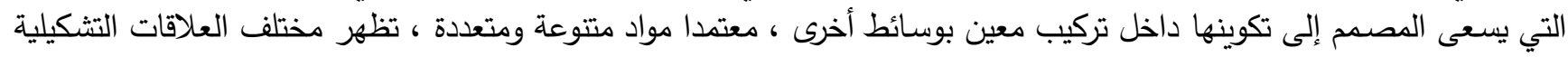

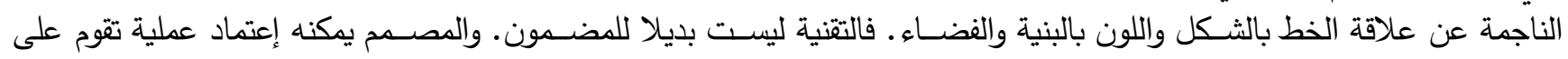

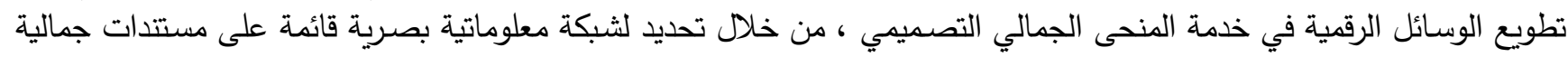

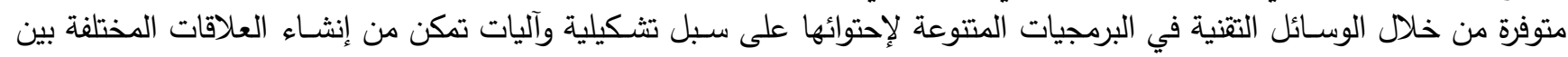

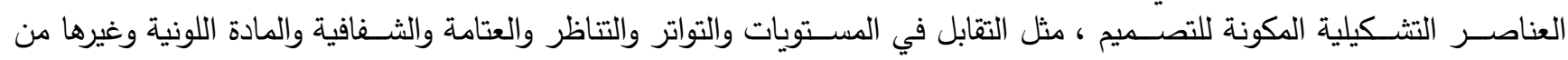

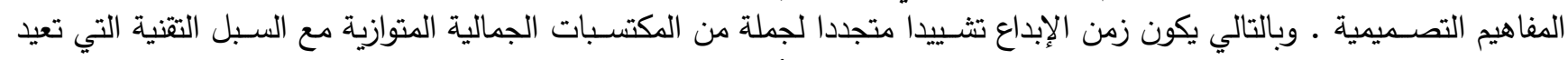

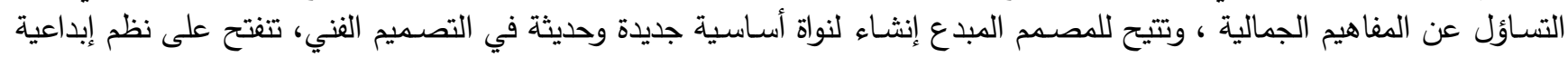

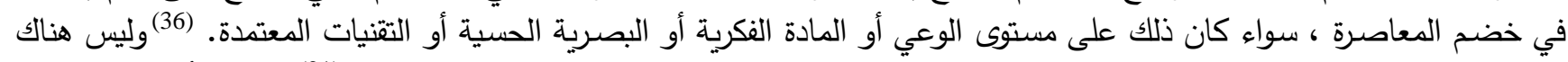

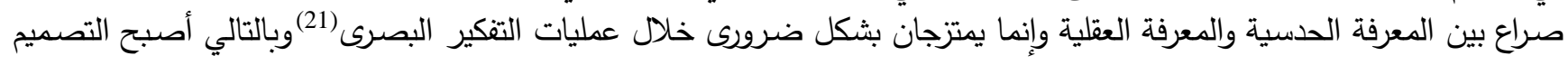

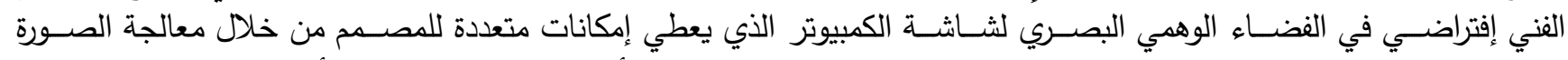

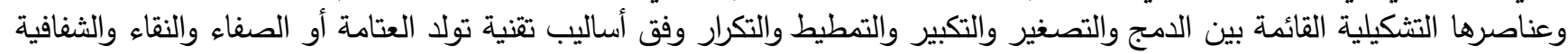

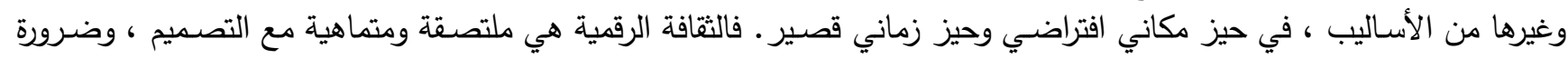

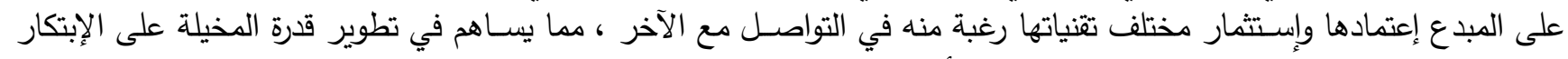

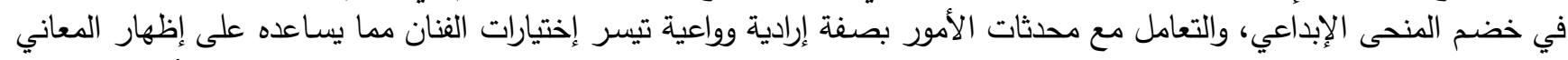

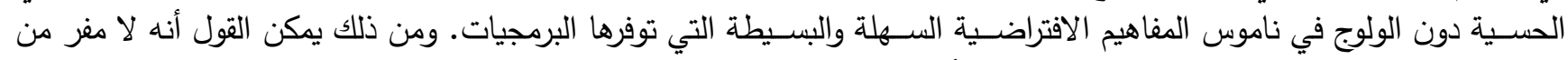

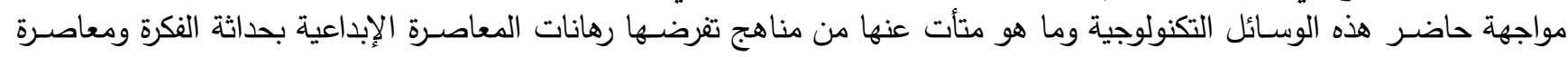

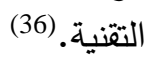

خمائن الفكرة الإبداعية: 1 ) تجذب الإنتباه .

2) تصلح للتطبيق بما يسمح باختباه بارها وتوافر عناصر بها يسمح بتقيمها وقياس فاعليتها.

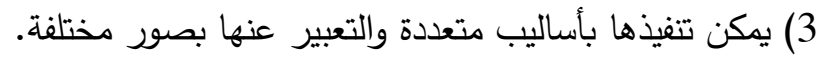

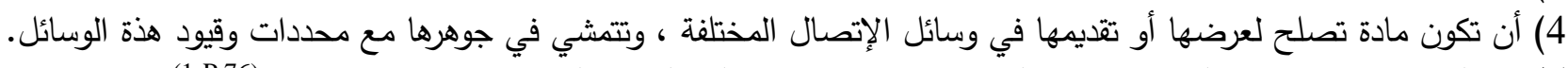

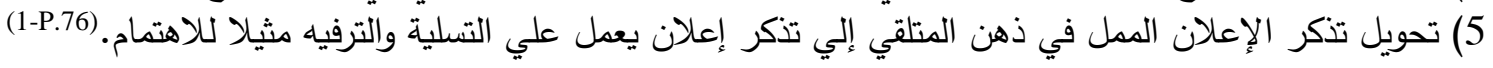

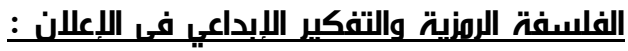

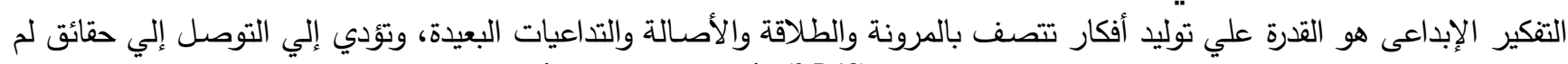

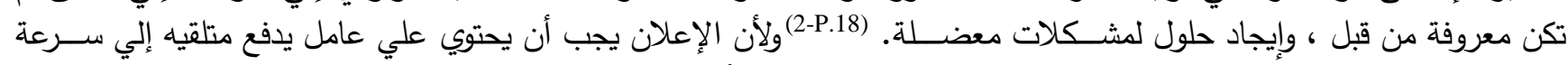

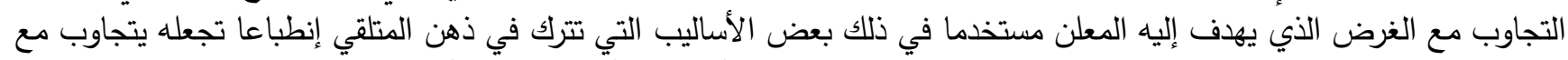

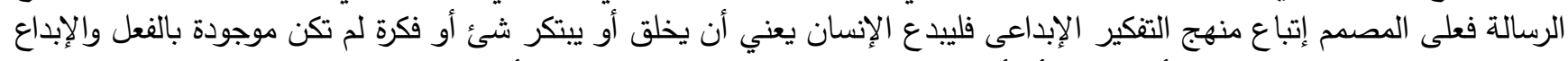

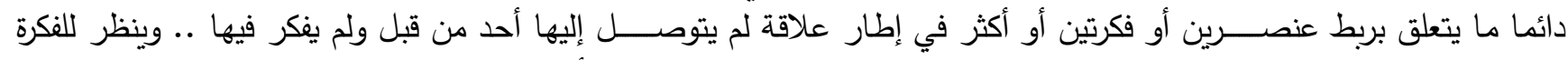

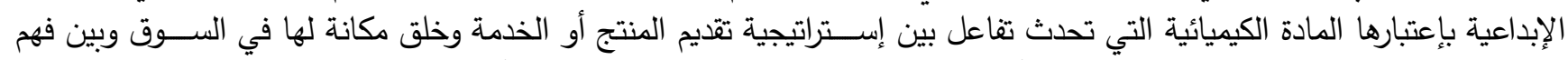

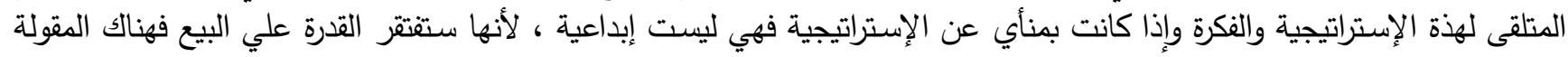

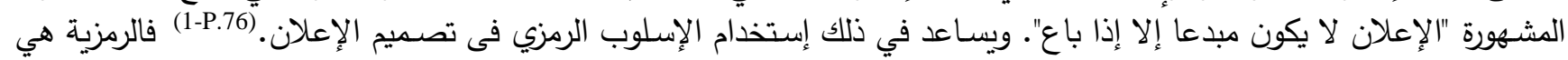

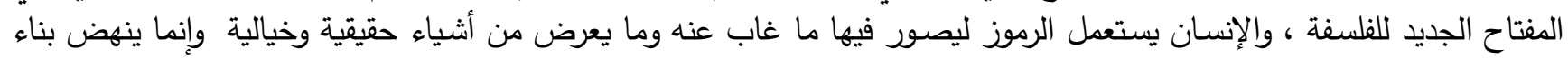


المعرفة الإنسانية ، لا من حيث أنه جملة معلومات حسية ، بل من حيث أنه تركيب من الحقائق التي هي بسبيل الرموز والقوانين التي

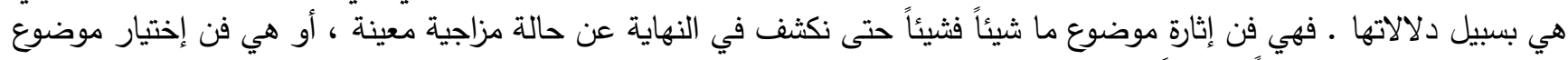

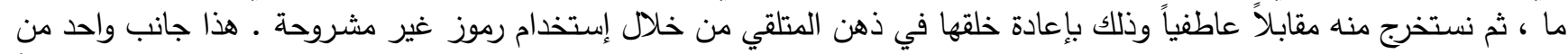

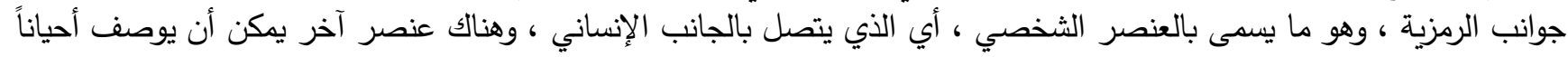

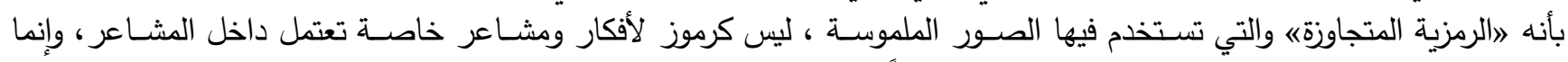

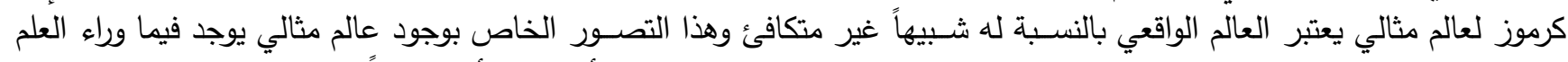

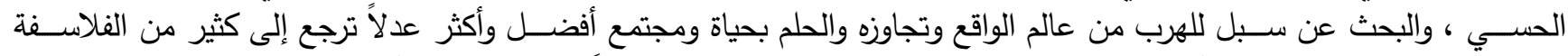

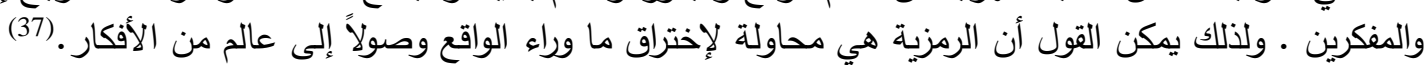

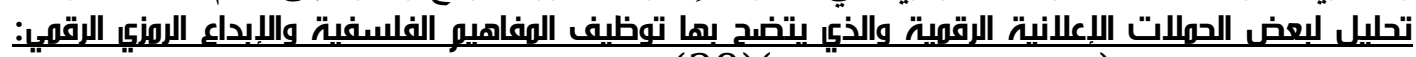

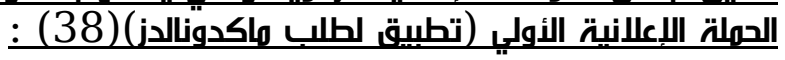
حملة إعلانية للتعريف عن اللفاليـ خدمة جديدة وهي تطبيق عبر الهاتف لطلب ماكدونالدز وإستخدمت الأسلوب الرمزي حيث إستبدلت أنواع

\section{McDonald's}

in : Toronto Agency Network: Cossette

Canada

Art Director: Simon Hung Advertising Agency: Cossette, Toronto الطعام بالهواتف المحمولة.

Mobile Ordering Here Posted: November 30, $2017 \quad$ Posted :Credits الوسيلة الأولى : (إعلانات منيثقة

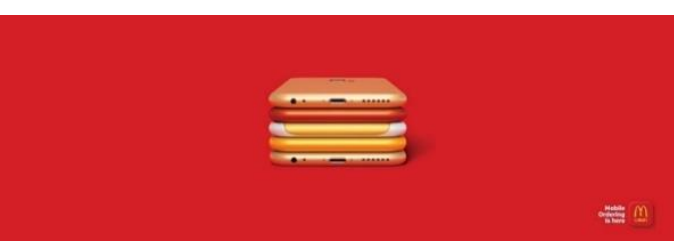

نموذج (3)

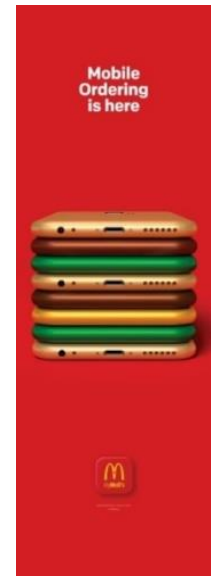

نموذج (2)

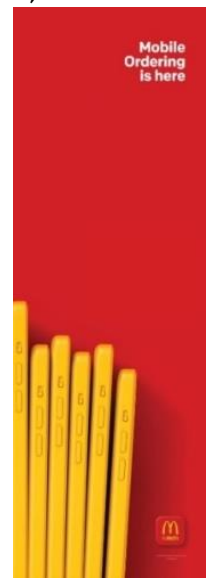

نموذج (1)

الوسيلة الثانية : (إعلانات مواقو توامل الإجتماعيا (Social Media Advertising "Faceook")

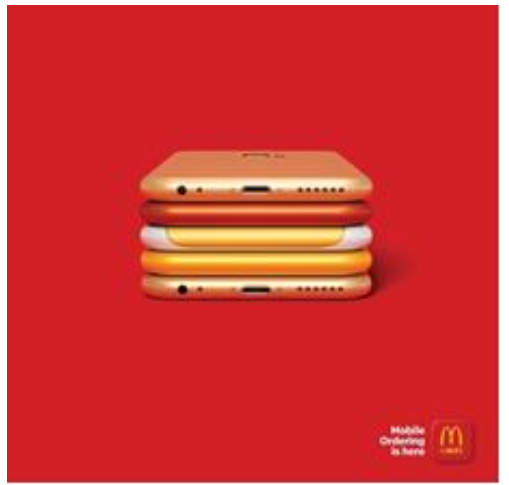

نموذج (5)

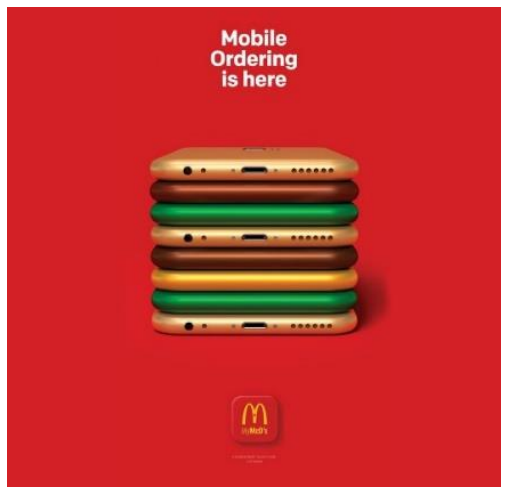

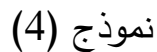

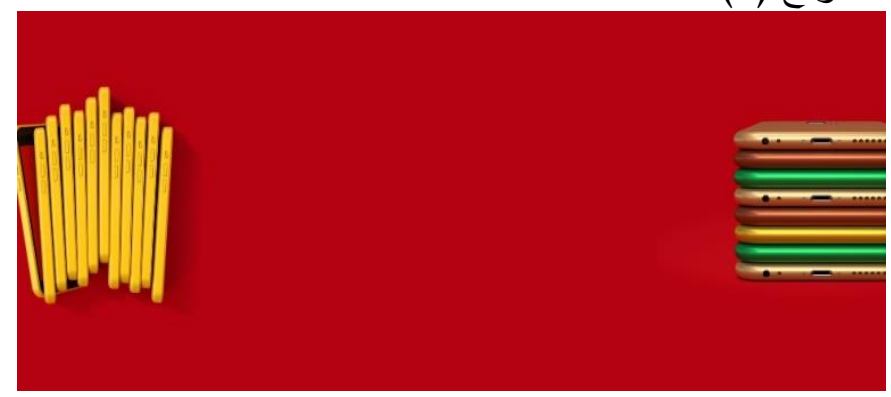

نموذج (6) 


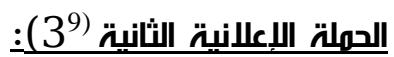
حملة إعلانية للتعريف عن خدمة جديدة وهي تطبيق عبر الهاتف لطلب ماكدونالدز وإستخدمت الأسلوب الرمزي حيث إستبدلت أنواع "Happy Moving Day"
in : Canada Agency Network: Cossette Posted: Monday, July 1 Posted الطعام بالهواتف المحمولة. المبن. :Credits

Advertising Agency: Cossette Montréal Art Director: Simon Caspar/David Theroux
Creative Directors: Janine Hopkinson Copywriter: Alexandre Emond-Turcotte

الوسيلة الأوليا: (إعلانات منبثقة Pop up Ads):

ن

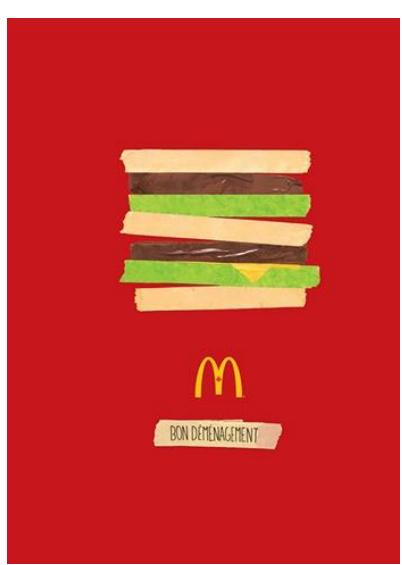

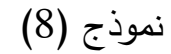

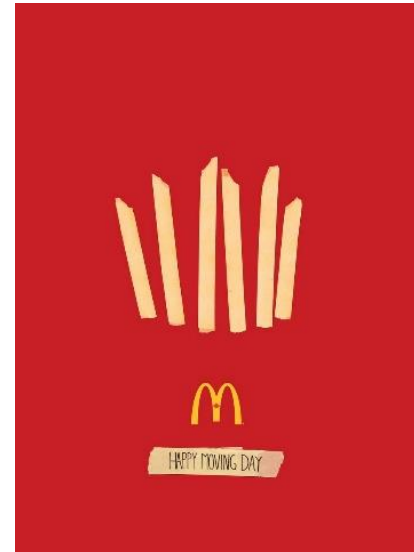

(7)

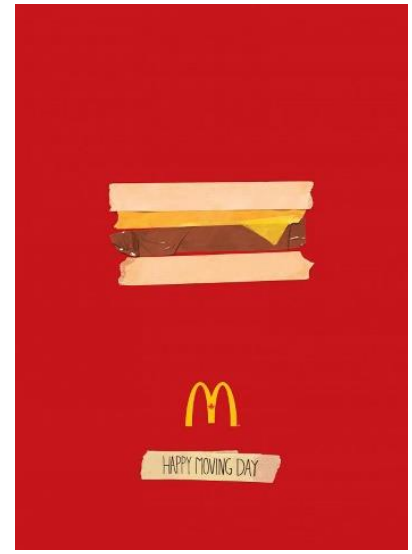

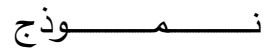

الوسيلة الثانية : (إعلانات مواقع التواصل الإجتماعيا Social Media Advertising "Faceook"

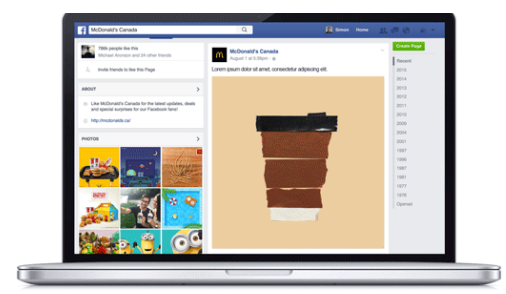

نموذج (10)

الحملة اللعالانية الثالثة (40):

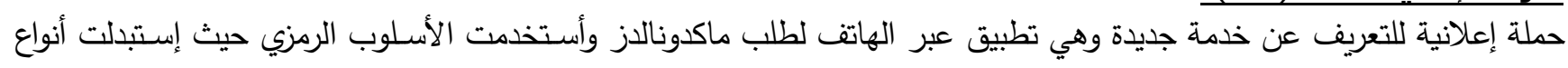
" Happy Moving Day" in : Canada Posted Agency Network: Cossette الطعام ببانتون الطلاء. McDonald's Posted: October 04, 2017 :Credits Creative Directors: Vicky Morin Advertising Agency: Cossette Montréal Art Director: Fabrice Bouty, Alexandre Jutras الوسيلة : (إعلانات منيثقة Pop up Ads) 


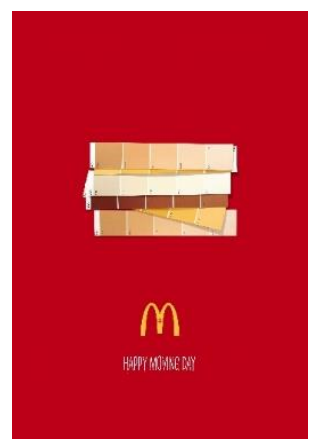

نموذج (13)

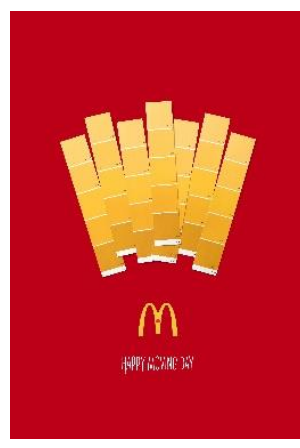

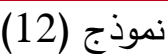

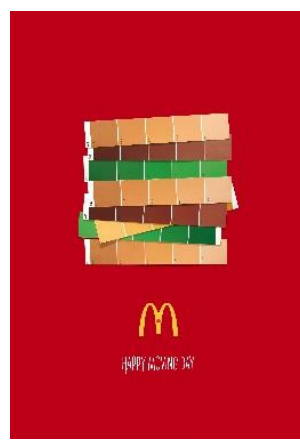

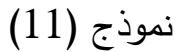

الحملة اللبعانية الرابعة (41):

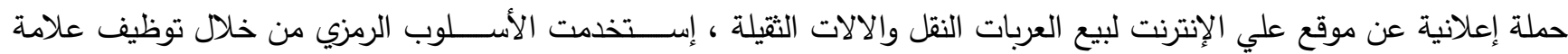

Tradus.com Search Bars - Tractor in : Germany Published/Aired: January 2019

محرك البحث علي شكل العربات .

Agency Network: Proximity Posted: March 01, 2019

Posted :Credits

Advertising Agency: Proximity, Duesseldorf, Germany Creative Directors: Nuno Melo Cristino, Sebastian Steller

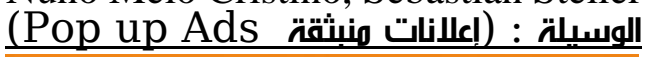

\section{Dump}

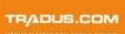

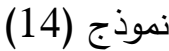

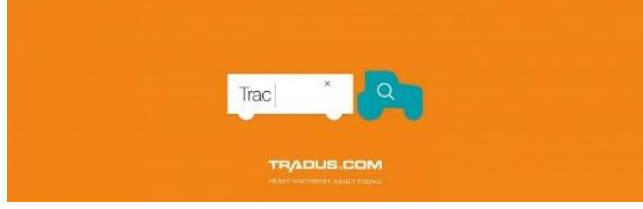

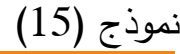

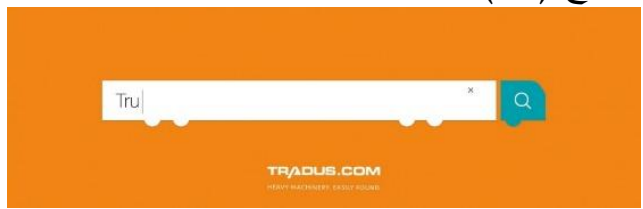

نموذج (16)

الحملة اللعالانية الخامسة: حملة إعلانية للتعريف عن العروض الإستة المقدمة من شـركة أيكيا بمناسبة رأس السـنة وإسـتخدمت الإسـلوب الرمزي في توظيف عناصـر

IKEA

Agency Network: TBWA

in : Portugal Posted: November 05, 2014
الإحتفال برأس السنة . لاتعريف عان

Early Christmas sale, Published/Aired: October 2014

Posted

Advertising Agency: TBWA, Lisbon, Portugal

Art Director: Julliano Bertoldi

الوسيلة الأوليا(42): (إعلانات منبثقة Pop up Ads) 


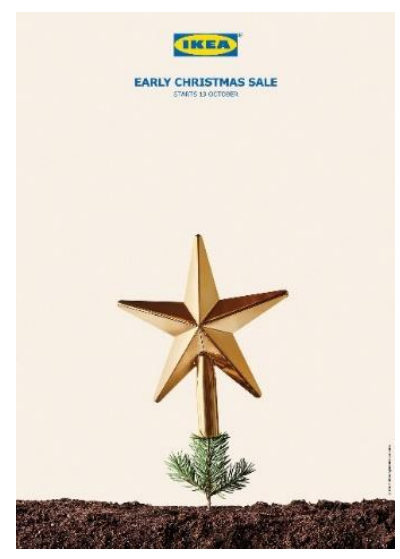

نموذج (18)

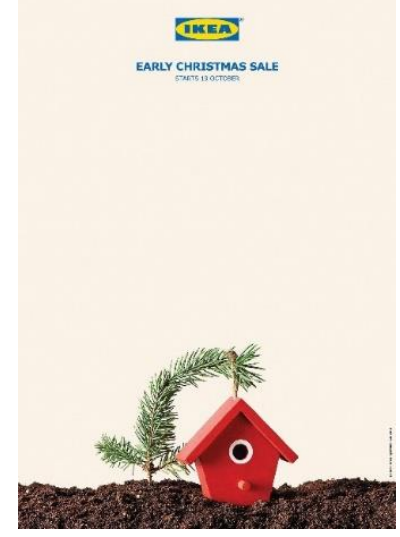

نموذج (20)

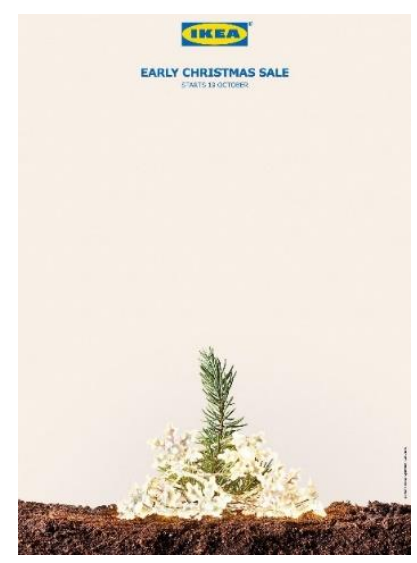

نوذج (17)

EARIY CHRISTMAS SALE

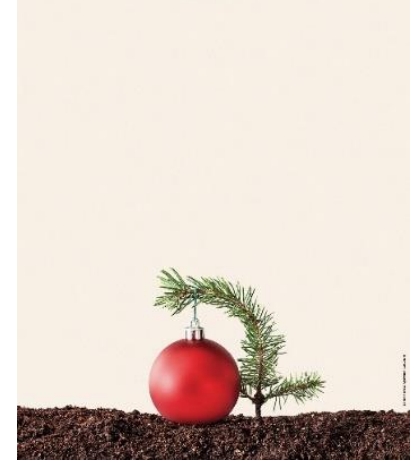

نموذج (19)

(Social Media Advertising "Faceook" الوسيلة الثانية (43) : (علانات مواقع تواملل اللجتماعيا

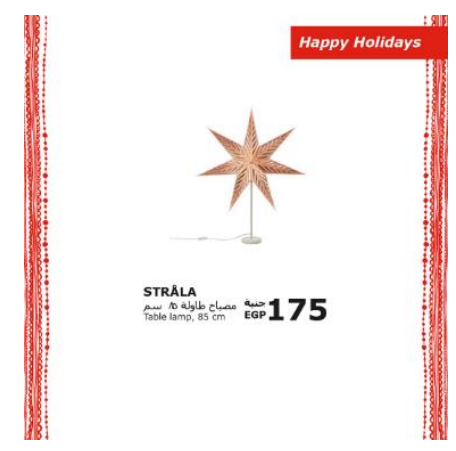

نموذج (23)

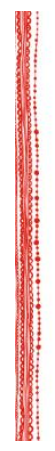

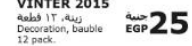

نموذج (22)

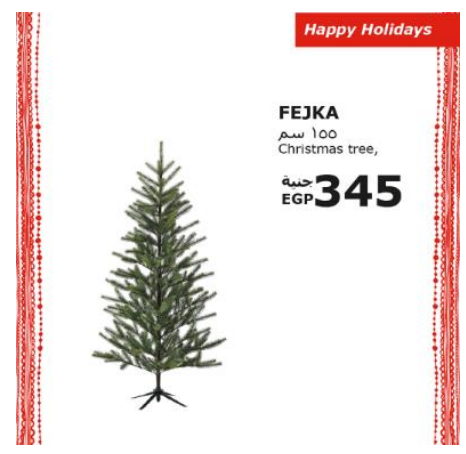

نموذج (21)

نتالح البحث:

1) بتحويل الدفاهيم الفلســفية الرمزية إلى عملية تطبيقية تتفتح نظم الإبداع البصــرى الرقمى والتى تعمل على تخطيط حملة إعلانية معاصرة ناجحة.

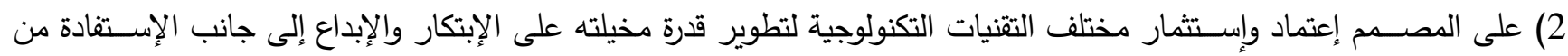

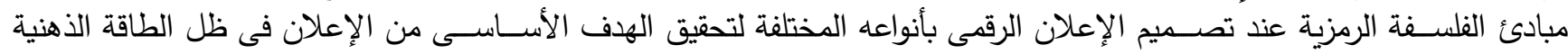
للمتلقى. 
3) الإبداع البصــرى الرقمى هو النثـاط الذهني الذي يحرك مجموع الفنون التى تولدت مع توظيف التكنولوجيا أو تطورت من أشـكال

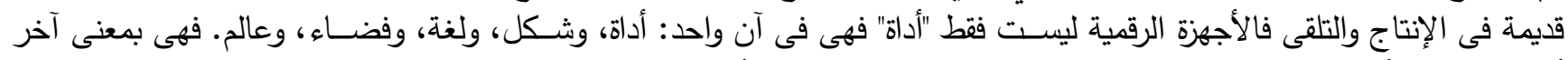

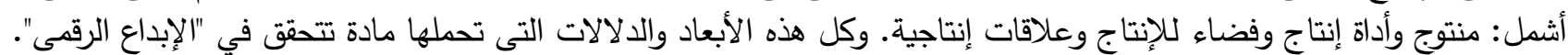

:

1) هذا النوع من الفنون الحديثة لا يمكن أن يتأسـسـ داخل أي مجتمع دون جهود حكومية واعية لإنثــــاء بنية تحتية متينة تتيح

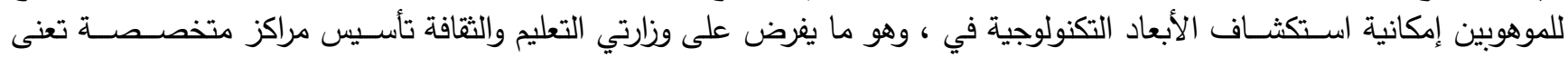

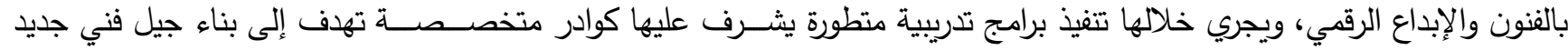

ينطلق من الأجهزة الذكية لإنجاز أفكاره المبتكرة.

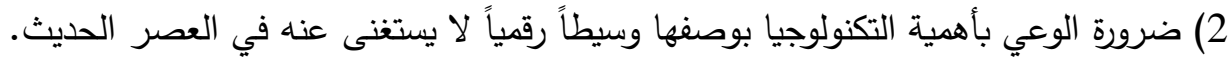

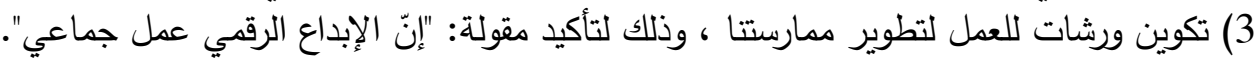

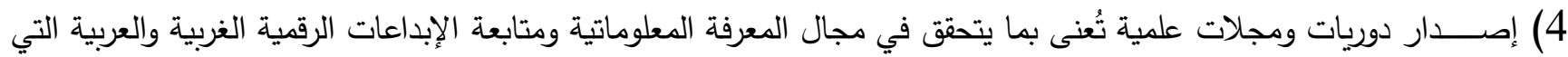

تصدر على شبكة الإنترنت.

:

الكتب العلمية:

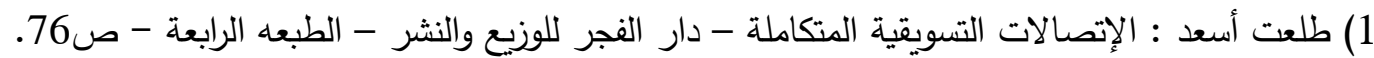

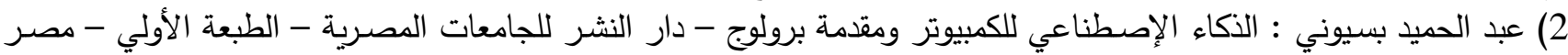

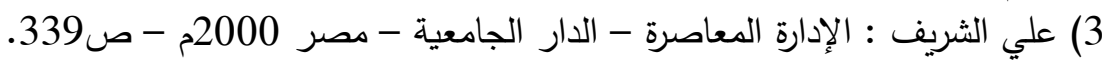

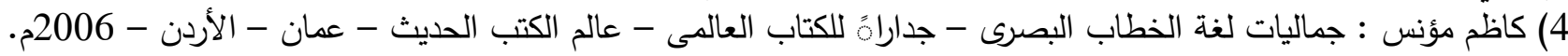

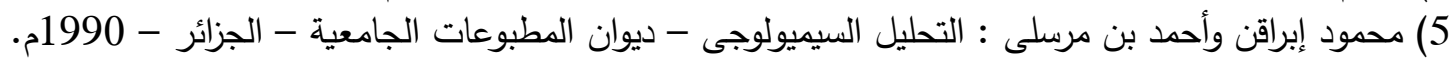

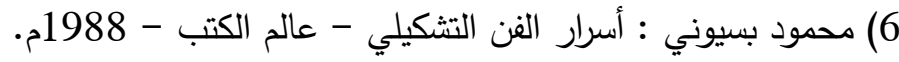

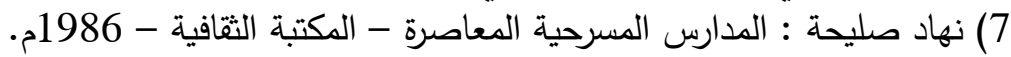

8) نور برت وينز : (السييرنطيقا التحكم والإتصال فى الحيوان والآلة)- الطبعة الثانية- كمبردج ماس 1948- 1961م.

الإسائل العلمية:

أولاً: رسائل الماجستير:

9) أمينة محمد علي نوار المصري : جمالية الرمز في فنون الحداثة وما بعد الحداثة - رسالة ماجستير - كلية التربية الفنية -جامعة

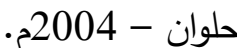

10) سارة جمال عبد القادر : دور الواقع الإفتراضي في بناء الأفكار الإعلانية - رسالة ماجيستير - كلية الفنون التطبيقية - جامعة حلوان - 2015م.

ثانياً: : رسائل الدكتوراه:

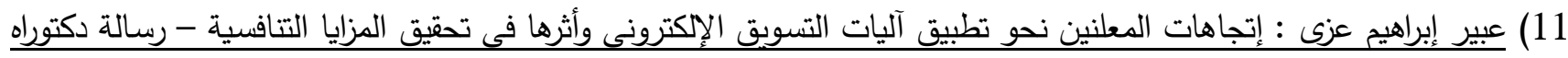

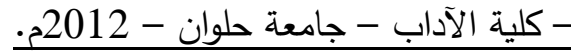

12) لمياء عبد الكريم قاسم : دلالات التصديم الجرافي التيكى في تصميم إعلانات التليفزيون المقدمة للطفل المصرى - رسالة دكتوراه كلية الفنون التطبيقية - جامعة حلوان - 2001م.

13) محمد حسين علي محمود الصبان : النسق الجمالي والمداخل المفاهيمية للإعلان في الفن المصـري القديم - رسالة دكتوراه -

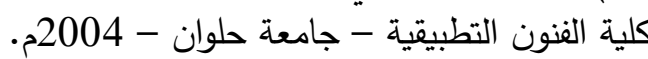

14) هبه أمير أحمد : الأبعاد الفلسفية للفنون ما بعد الحداثة وتطبيقاتها لتجسيد الواقع الإفتراضسى فى الإعلان المعاصـر - رسـالة

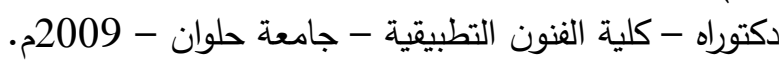

15) هدى عثمان محمد : رؤية معاصـرة للفلسـفة الرمزية فى تصــيم الإعلان الحديث - رسـالة دكتوراه - كلية الفنون التطبيقية -

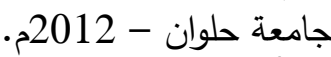

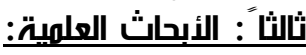

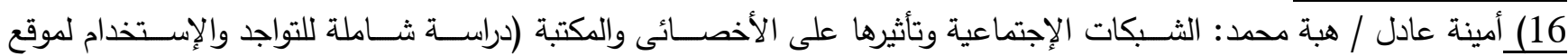

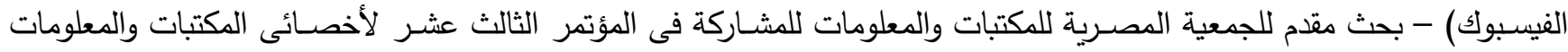

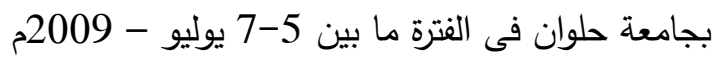


17) محمد يســرى عبد الغنى/ عبد النبى أبو الدجد / محمد عبد الحميد محمد : دور العوامل الأرجونوميكية والبيئية فى إبتكار

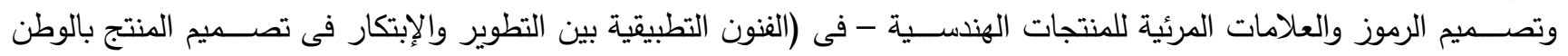

العربى) - المؤتمر العلمى لكلية الفنون التطبيقية - جامعة حلوات المنان - 2002م.

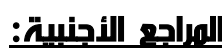

18) Daniel Chandler: (Semiotics: The Basics) $-2^{\text {nd }}$ edition - Taylor \& francis Group - London - 2007. 19) Golob, Thomas F. and Amelia C. Regan (2001) "Impact of Information Technology on Personal Travel and Commercial Vehicle Operation: Research Challenge \& Opportunities". Transportation Research, 9.

20) Shar Vanboskrik and Emily Riley - The Future of Interactive Marketing - Forrester Research, Inc

21) Willman R.E - Visual Communication - Englewood Cliffs.w.g - educational technology publication. USA. 1993.

مواقع الإنترنت:

22)

https://www.google.com.eg/search?hl=en-

EG\&q=screenshot\&tbm=isch\&tbs=simg:CAQSlwEJD75 cLLE4PacaiwELEKjU2AQaBAgVCAo

MCxCwjKcIGmIKYAgDEiiNCLgTuhOKCLITtxOzE7sT_1AeuE-8_18D_1XP6M-

rz7uP6Urqii8PokrGjCLuetLPYonz9KNQeuehm8w3bh-

f_1AqdazZJAfTftI_1bIgkVoYRhR8M4zaMioHL7uwgBAwLEI6u_1ggaCgoICAESBHFMV9UM

\&sa=X\&ved=0ahUKEwjQ2Zvtq-

3gAhVKTxUIHZmfB AQwg4IKCgA\&biw=1366\&bih=608\#imgrc=LoNBCxq1_tSK6M

23) https://plus.google.com/104017314171836070647/posts/Jg8BQYLdoNn

24) http://obelismedia.com/social-media-advertising/

25) https://www.outbrain.com/native-advertising /

26) https://www.wordstream.com/pay-per-click-advertising

27) https://www.perion.com/blog/8-types-online-advertising-need-know/

28) https://en.wikipedia.org/wiki/Pay-per-click

29) https://en.wikipedia.org/wiki/Behavioral_retargeting

30) https://blog.bannersnack.com/flash-banners-vs-gif-banners-vs-static-banners/

31) https://99designs.com/blog/web-digital/animated-alternatives-flash-banners/

32) https://searchcrm.techtarget.com/definition/floating-ad

33)https://www.google.com/search?rlz=1C1SQJL_enEG831EG831\&biw=1366\&bih=657\&tbm= isch\&sa=1\&ei=3aJ XOn9JOnUgwfk9r3wDQ\&q=Audience+view++for+the+Internet+ad+and+t $\mathrm{v}+\mathrm{ad}+\mathrm{in}+2018 \& \mathrm{kq}=$ Audience+view++for+the+Internet $+\mathrm{ad}+\mathrm{and}+\mathrm{tv}+\mathrm{ad}+\mathrm{in}+2018 \& \mathrm{gs} \_\mathrm{l}=\mathrm{img} .12 \ldots$ $121906.122697 . .124076 \ldots 0.0 . .0 .146 .265 .0 \mathrm{j} 2 \ldots \ldots .0 \ldots .2 \mathrm{j} 1 . . \mathrm{gws}-\mathrm{wiz}-$

img.nIA_1P2q8Zw\#imgrc=fxTjK50DbbBtjM:

34) http://www.m.ahewar.org/s.asp?aid=164653\&r=0

35)https://www.mominoun.com/articles/\%D8\%A7\%D9\%84\%D9\%83\%D8\%AA\%D8\%A7\%D8

\%A8\%D8\%A9-\%D8\%A7\%D9\%84\%D8\%B1\%D9\%82\%D9\%85\%D9\%8A\%D8\%A9-

\%D8\%B9\%D9\%86\%D8\%AF-\%D8\%B3\%D8\%B9\%D9\%8A\%D8\%AF-

\%D9\%8A\%D9\%82\%D8\%B7\%D9\%8A\%D9\%86-2988

36)http://www.essahafa.tn/wordpress/2013/04/24/\%D9\%85\%D9\%82\%D9\%88\%D9\%85\%D8\%A

7\% $8 \%$ AA-\%D8\%A7\%D9\%84\%D8\%A5\%D8\%A8\%D8\%AF\%D8\%A7\%D8\%B9-

\%D9\%81\%D9\%8A-\%D8\%A7\%D9\%84\%D9\%81\%D9\%86-

\%D8\%A7\%D9\%84\%D8\%B1\%D9\%82\%D9\%85\%D9\%8A/

37)https://www.alroeya.com/article/8646/\%D8\%A7\%D9\%84\%D8\%B1\%D9\%85\%D8\%B2\%D9 $\% 8 \%$ D $8 \%$ A9-\%D9\%81\%D9\%8A-

\%D8\%A7\%D9\%84\%D9\%81\%D9\%84\%D8\%B3\%D9\%81\%D8\%A9

38) https://www.redflagdeals.com/latest-news/17/11/30/mcdonalds-mobile-ordering-is-nowavailable-in-toronto/

39) https://www.infopresse.com/dossier/2016/2/11/grand-prix-demenagement-par-cossetteaffiche-publicitaire

40) https://www.adsoftheworld.com/media/print/modonalds_breakfast

41)https://www.adsoftheworld.com/media/outdoor/traduscom_search_bars_tractor

42)https://www.adsoftheworld.com/media/outdoor/ikea early christmas sale 1

43) https://www.facebook.com/IkeaEgypt/photos/a.731900726953945/732255560251795/?type $=3$

\&theater 